\title{
Atomistic Theory and Simulation of the Morphology and Structure of Ionic Nanoparticles
}

Dino Spagnoli and Julian D. Gale

Nanochemistry Research Institute, Department of Chemistry, Curtin University, PO Box U1987, Perth, WA 6845, Australia

\begin{abstract}
Computational techniques are widely used to explore the structure and properties of nanomaterials. This review surveys the application of both quantum mechanical and force field based atomistic simulation methods to nanoparticles, with a particular focus on the methodologies available and the ways in which they can be utilised to study structure, phase stability and morphology. The main focus of this article is on partially ionic materials, from binary semiconductors through to mineral nanoparticles, with more detailed considered of three examples, namely titania, zinc sulphide and calcium carbonate.
\end{abstract}

\section{Introduction}

Nanoscience and nanotechnology collectively represent one of the fastest growing interdisciplinary scientific areas, spanning interests from physics, through chemistry and geoscience, to biology. Be it the electronic or mechanical properties of a material, once the key dimension(s) of the system reaches the critical size, typically in the range of 1$100 \mathrm{~nm}$, then the characteristics exhibit a size-dependent variation. Thus size becomes a new design parameter alongside chemical composition. This paradigm shift has created new opportunities in fields including solar energy, ${ }^{1}$ electronics, ${ }^{2}$ medical therapies, ${ }^{3}$ energy storage, ${ }^{4}$ and catalysis. ${ }^{5}$ Indeed the commercial value of items containing nanotechnology was estimated to be US\$250 billion for 2009 . $^{6}$ 
One of the greatest challenges of the nanoscale is our ability to fully probe the atomic details of structure and properties. Although experimental techniques, such as atomic force and scanning tunnelling microscopy, ${ }^{7-9}$ can potentially provide images with sufficient resolution, care must be taken in interpreting what is observed. Because of such difficulties, nanoparticles and nanomaterials have been a fertile ground for theoretical studies to support experiment, and in some cases even lead the way. The recent literature has seen an explosion in the application of modelling techniques to nanoscale objects, both real and hypothetical. Of course, there are also molecular inorganic objects that have dimensions of a nanometre or greater that represent ideal model systems for study where the structure is well known. ${ }^{10}$

Broadly speaking, the study of nanostructured materials can be divided into several main categories including metal nanoparticles, carbon nanomaterials and their analogues, semiconductor quantum dots, and ionic materials. There are of course arguably many other sub-divisions, such as covalent molecules, colloids and nanoporous frameworks that can have critical dimensions in the range of $1-100 \mathrm{~nm}$ and so any attempt to rigorously define categories will be flawed. Due to the extensive nature of the nanomaterials literature, the aim of this article is not to attempt to review everything. Instead, the focus will be on highlighting what can be achieved through the use of theory and simulation in the characterisation of nanoparticles for primarily one particular type of system, namely ionic compounds. Reviews of the literature for carbon nanomaterials ${ }^{11-13}$ and metal clusters ${ }^{14-16}$ can be found elsewhere. However, some comparisons will be drawn between the techniques applied to each type of system.

In this review we will first examine some of the methodologies that are prevalent in the literature for the simulation of nanoparticles, in order to highlight the state of the field and current trends. The application of these techniques will then be demonstrated by reviewing studies of a few key examples of at least partially ionic nanomaterials, including titania, zinc sulphide and calcium carbonate. 


\section{Methodologies for nanoparticle simulation}

Simulation methods for the study of bulk materials have become well established over the last half a century, in parallel with the advances in computing. The use of periodic boundary conditions permits the modelling of a quasi-infinite solid to be performed routinely with a complexity that is related to a few symmetry-unique atoms. Even the simulation of surfaces has become routine by employing a 2-D infinitely periodic description (though this is often mapped back to 3-D with a gap between repeating surfaces for computational convenience). ${ }^{17}$ When considering nanoparticles, the scenario is quite different due to the high surface area to volume ratio. Surfaces can no longer be considered independent, especially in ionic materials where electrostatic interactions couple terminations over large distances. Furthermore, the energetics of edges and corners can become a driver for particle morphology too. To further complicate matters, the perturbation due to the particle termination can be large enough to induce a phase transformation, such that the underlying structure of the material differs from the bulk at the nanoscale. Add to this the fact that nanoparticles must be supported or contained within some chemical environment to prevent collapse and the full challenge of predictive nanomaterials modelling can be appreciated.

Before engaging in the more specific discussion of simulation of nanomaterials, a brief introduction to what an atomistic simulation actually involves may be in order. The problem of simulation involves characterising the energy landscape for an assembly of atoms. Typically the starting point is to consider the potential energy surface for a system, though ultimate the relative free energies of different configurations are the objectives. If a potential energy surface has well-defined minima, separated by barriers substantially larger than ambient thermal energy, then the main task of a simulation is to locate these states and determine their properties. For ordered materials, the use of optimisation to follow the energy surface downhill to the nearest basin can be sufficient. The challenge is always to ensure that the lowest energy minimum has been found. For more disordered or flexible systems, such as liquids or amorphous materials, the properties must be obtained by statistical sampling of all of the thermally accessible minima within a given ensemble. Here two classes of method are commonly used: 
1) Monte Carlo (MC) sampling can be employed where the system propagates through random displacements and the running average of computed properties, weighted by the probability of the configuration occurring given by the Boltzmann factor, is used to approximate the integral over the ensemble. ${ }^{18}$ This technique can be improved in efficiency through the use of importance sampling, whereby the Boltzmann factor becomes the acceptance criterion for a trial move, thus driving the system to primarily explore those configurations that contribute most to the ensemble average. The advantages of Monte Carlo as a technique are that it only requires the energy to be calculated, rather than the forces, at each atomic configuration, and that it is simple and efficient to implement, including on parallel computers.

2) Molecular dynamics (MD) similarly moves across the potential energy surface sampling configurations, but this is now performed according to Newton's equations of motion. ${ }^{19}$ Here the atoms are typically treated as classical particles with a given mass and velocity. They then accelerate according to the force they experience and migrate across the potential energy surface. The initial velocities are typically drawn from a MaxwellBoltzmann distribution and then scaling or a thermostat is used to achieve a target temperature in an isothermal ensemble. Because time must be advanced in discrete steps to perform the numerical integration of the particle motion, the degree of sampling achieved is limited by the time step (often of order $\sim 1$ fs $=1 \times 10^{-15} \mathrm{~s}$ ) and the total number of steps executed. The advantage of molecular dynamics over Monte Carlo is that can, in principle, provide information regarding the rates of fast processes, as well as the thermodynamic averages. However, because atomistic simulation lengths vary between a few picoseconds, when using quantum mechanical forces, up to a millisecond, when based on more approximate forces, molecular dynamics can be trapped in a region of the potential energy surface if the barrier to escape is too high.

There are other techniques and variants of these two categories of simulation method, such as genetic algorithms, simulated annealing, etc, most of which are designed to 
increase the degree of exploration of the potential energy surface, often in search of the global minimum. Such techniques are particularly important for clusters and nanoparticles where the structure may differ from that of the corresponding bulk. More information can be found regarding different simulation approaches elsewhere.

Ultimately the objective of simulating nanomaterials is often to understand the structure-property relationship so that control can be exerted over the system to exploit the unique effects of nanoconfinement for technological benefit. In some cases this may not require detailed atomic-scale modelling. For example, the modelling of confinement of electronic states in semiconductor quantum dots can be achieved based on solving Schrödinger's equation for electrons in a model potential that reflects the nature of the device structure. This effective mass approximation ${ }^{20}$ contains empirical parameters, but is generally successful in predicting the variation of confinement effects with device structure. ${ }^{21}$ Similarly, the diffusive behaviour of nanoparticles can be simulated using mesoscale modelling techniques, such as dissipative particle dynamics (DPD), in which atoms or molecules are grouped together as beads on which collective forces act. Yang and $\mathrm{Ma}^{22}$ have used this approach to explore how nanoparticle shape and angle of incidence influence the ability of such species to cross a lipid bilayer. This is an important first step to understanding the effectiveness of nanoparticles for drug delivery across cell membranes. A similar approach was previously used $^{23}$ to examine nanoparticle interactions with vesicles, where they were shown to cause a change in the structure of the organic material, albeit in the absence of an explicit solvent. Monte Carlo simulation of the agglomeration of nanoparticles has also been performed within classical DLVO theory. ${ }^{24}$ Here the nanoparticles are approximated as a sphere containing a dipole moment within a dielectric medium, where the dielectric constant sharply decreases as two particles come into contact. This coarse-grained description is sufficient to capture the tendency of nanoparticles to spontaneously self-assemble into structures that exhibit degrees of chain-like character. Refinement to include particle dipole moments based on semi-empirical calculations for CdTe leads to similar behaviour during assembly. ${ }^{25}$

Intrinsic properties of individual nanoparticles may also be probed without specific reference to full atomic complexity. For example, in the case of metal nanoparticles the plasmon resonance is often the quantity of interest. The variation of this quantity with 
nanoparticle anisotropy can be modelled using the discrete dipole approximation (DDA). ${ }^{26-28}$ Here the metal particle adsorption spectrum is determined from the response properties of the self-consistent solution for a grid of coupled polarisable centres with induced dipoles. In the case of ionic nanoparticles, such as those of transition metal oxides, other properties can be simulated by a similar grid site treatment. Instead of an induced dipole, the on-site quantity is now an electron spin of fixed magnitude, but variable orientation. By either energy minimisation or Monte Carlo solution of a Hamiltonian involving the exchange interaction, typically between only neighbouring sites, the magnetisation of the nanoparticle can be found. This approach has been applied to binary phases, such as maghemite $\left(\gamma-\mathrm{Fe}_{2} \mathrm{O}_{3}\right),{ }^{29,30}$ and to ternary materials, especially spinels. $^{31}$

Although it is possible to simulate a number of properties or behavioural trends of nanoparticles without recourse to the specific atomic details, beyond composition, ultimately a full understanding can require some form of atomistic simulation. Hence, we now consider some of the methodologies that have been adopted in this category. In general terms, there are two classes of atomistic approach - those that explicitly treat the electronic degrees of freedom and those that try to capture the potential energy surface with only implicit regard for electrons. Typically the former techniques involve quantum mechanical solution of some form of wave equation, whereas the latter can be broadly referred to as force field methods. That said, the division is far from clear-cut since increasingly force fields include contributions from charge-transfer, ${ }^{32}$ dipolar or quadrupolar polarisability ${ }^{33}$ or even ligand-field effects, ${ }^{34}$ all of which involve electronic degrees of freedom that go beyond nuclear position. Furthermore, methods such as empirical valence bond theory use a coupling Hamiltonian to mix two or more classical force field potential energy surfaces, thereby spanning both categories. ${ }^{35}$

The most rigorous atomistic approach to calculating the properties of nanomaterials is using quantum mechanical methods. Wavefunction theory (WFT) offers the best way to systematically improve the accuracy of calculations through post-Hartree-Fock techniques, such as coupled cluster theory, configuration interaction, multi-reference methods and perturbation theory. However, given the size of most nanostructures, the application of these techniques has rarely been practical to date. Consequently, the vast 
majority of studies have focused on the use of Kohn-Sham density functional theory (DFT). Although not systematically improvable in a guaranteed manner, there have been several refinements in recent years that have improved the quality of calculations, especially in the condensed phase limit. New forms of the Generalized Gradient Approximation have emerged, such as the functionals AM05, ${ }^{36} \mathrm{Wu}$ and Cohen, ${ }^{37}$ and PBEsol $^{38}$ that typically offer a better description of solid-state properties, though often at the expense of other quantities, including atomisation energies of molecules. Hybrid functionals, ${ }^{39}$ that mix in a fraction of Hartree-Fock exchange, have long been available for molecular systems are now becoming more widespread within periodic solid-state programs, though with a substantially increased computational cost. Screened hybrids can represent a good compromise that trade off the local benefits of improved exchange against the increased calculation time. ${ }^{40}$ A well-known failing of DFT is the lack of longrange dispersion forces, leading to difficulties in describing weak interactions. For instance, most GGAs fail to capture the attraction between two graphene layers something that is particular problematic when studying assembles of carbon nanomaterials. This limitation can now be addressed through either the addition of an explicit damped dispersion interaction, in the style of a force field correction, ${ }^{41,42}$ or through use of truly non-local functionals (i.e. ones that depend on the density and its gradient at two points in space simultaneously). ${ }^{43}$ These approaches have various merits and demerits; the former method is simple to implement and inexpensive to calculate, while the later is less empirical since the functionals are universal. Following the development of efficient algorithms, ${ }^{44}$ the use of non-local van der Waals functions need not lead to a substantial extra computational cost.

One of the motivations for the use of quantum mechanical methods in the study of nanoparticles, aside from obtaining reliable systematic energetics, is to probe the electronic properties. Densities of states for nanoparticles are routine by-products of a DFT calculation. However, these Kohn-Sham orbitals are not genuine single particle states due to self-interaction error within actual exchange-correlation functionals. ${ }^{45}$ To compute a more reliable electronic adsorption spectrum, the dominant approach for nanomaterials is to employ time-dependent density functional theory (TDDFT). ${ }^{46}$ Here both linear-response and real-time propagation approaches can be used, where the latter 
method has potential advantages for larger systems, ${ }^{47}$ as well as including higher-order contributions. As an example, this approach has been used to compute the polarisability and dipole strength of $\mathrm{C}_{60} \cdot{ }^{47,48}$ The interaction between adsorbed molecules and oxide nanoparticles, especially of those of titania, has attracted several quantum mechanical studies because of the interest in sensitisation of solar cells and biomedical applications. While some studies have considered just the Kohn-Sham states, ${ }^{49}$ there are an increasing number of studies that have applied TDDFT to titania clusters, ${ }^{50,51}$ including with adsorbed species. ${ }^{52-55}$

Because of the size and complexity of nanostructures, especially when including adsorbates or a surrounding environment, it can be advantageous to use more approximate forms of quantum mechanics by simplifying the Hamiltonian. Densityfunctional tight binding (DFTB) is one such method that has been applied to the study of nanomaterials. ${ }^{56-58}$ Semi-empirical quantum mechanical methods, such as MNDO-based techniques, are also applicable in many cases, such as $\mathrm{CdS}$ nanoparticles and their capping, ${ }^{59,60}$ which have also been studied using DFTB. ${ }^{61}$

An alternative to reducing the complexity of the Hamiltonian by explicit simplification is to apply some degree of localisation to the matrix elements in order to lead to linear-scaling construction. This can be achieved by either neglecting integrals below a given threshold or by strict localisation of the basis functions. ${ }^{62}$ In this way, the scope of DFT electronic structure calculations can readily be extended to the order of a thousand atoms. Beyond this point, the computational cost becomes increasingly dominated by the time required to solve for the new orbitals and their eigenvalues, rather than the Hamiltonian construction. Even if the Hamiltonian is sparsely populated, the Kohn-Sham states can potentially be delocalised across all atoms, leading to a dense matrix solution with $O\left(N^{3}\right)$ scaling. In materials with a HOMO-LUMO gap, it is possible to solve the self-consistent field problem with algorithms that can achieve linear-scaling. Two such methods are functional minimisation ${ }^{63}$ and density matrix purification. ${ }^{64}$ Both algorithms allow the ground state density and energy to be determined, and so are suited to geometric relaxation of nanostructures, but provide no information regarding the electronic eigenvalue spectrum. An alternative strategy is to use a divide and conquer approach, ${ }^{65}$ which breaks the Hamiltonian down into a series of overlapping regions, 
coupled by a common Fermi energy. Although the orbital levels are now computed and available, any delocalised states will be most strongly perturbed by the confinement approximation and often these are the wavefunctions being sought. Unfortunately, from the point of view of achieving robust linear-scaling, the size of the HOMO-LUMO gap tends to decrease with increasing nanoparticle size making use of such techniques most difficult for the cases where they are most needed.

If only the energy and forces are required in order to explore the atomistic potential energy surface, rather than the full details of the electronic properties, then the use of force field methods can often be preferable, provided there is a reliable parameterisation. The choice of force field is usually very specific to the type of material to be studied. For metal nanoparticles, the use of the Embedded Atom Model (EAM) ${ }^{66}$ or modified variants ${ }^{67}$ are very much the de facto standards as they capture some of the many-body effects that are missing from a simple Lennard-Jones representation. For the majority of semi-conducting materials, the most widely applied model is that of Tersoff, ${ }^{68}$ while for the specific case of carbon nanomaterials there are a number of alternative formulations, including the Environmentally Dependent Interaction Potential (EDIP) ${ }^{69}$ Reactive Empirical Bond Order (REBO) ${ }^{70}$ and $\mathrm{LCBOP}^{71}$ models. On the face of it, this would seem to represent quite a disparate set of force fields, though all of them are based on the central tenant that the energy of an atom in a material is dependent on some function of the total bond order at that site. ${ }^{72}$ All that changes is largely the complexity of the expressions involved. Although widely used, most of the above models have limitations. For example, the REBO model has no long-range interaction and cannot describe binding between graphene sheets unless modified. ${ }^{73}$

Turning to the primary focus of this review, namely more ionic materials, the main force field approaches start with the dominant electrostatic contribution and then combine this with short-range repulsive terms, often of Buckingham or Lennard-Jones form. Some functional groups may exploit a molecular mechanics description within a fragment, e.g. for hydroxyl or carbonate anions, and occasionally angular terms are required, as in some models for silicates ${ }^{74}$ For anions, inclusion of dipolar, or even higher order, polarisability is important. One way to do this is to add point ion polarisabilities to the relevant sites. ${ }^{75}$ Alternatively, the shell model ${ }^{76}$ provides a simple mechanical description of dipolar 
polarisation that is naturally damped by the surrounding crystalline environment. Broadly speaking, force fields for ionic materials can be divided into those that adopt formal charges with inclusion of polarisability, and those that use unpolarisable partial charges. The latter are clearly advantageous for large-scale molecular dynamics since there is no need to handle the extra complexity of the quasi-electronic degrees of freedom in the equations of motion. However, this often comes at a price in terms of accuracy. For example, in the case of silica, all current partial charge models, including variable charge ones, give the wrong phase diagram, with $\alpha$-quartz not being the ground state. ${ }^{77}$

An exciting trend in force fields is the increasing development of so-called reactive force fields. Here we distinguish between reactive force fields and those that allow for smooth dissociation by requiring that the former can describe the breaking of covalent bonds and/or multiple oxidation states. Although some of the aforementioned models fall into this category, they have typically been limited to a small number of elements (e.g. hydrocarbons). The key to most reactive force fields is the inclusion of charge calculations on-the-fly by some form of electronegativity equalisation or local chargetransfer scheme, though there are exceptions. ${ }^{78}$ A number of different approaches have been derived in the materials science field with the aim of treating interfaces, especially those between metals and/or metal oxides. ${ }^{79-81}$ The power of this approach has been demonstrated through simulations of the oxidation of aluminium nanoparticles leading to the formation of a non-stoichiometric oxide shell surrounding the metallic core. ${ }^{82}$ The case of silicon-silica interfaces is also important for gate dielectric materials where the critical dimension is on the nanoscale, as well as silicon quantum dots embedded in silica. ${ }^{83-85}$

Arguably the most prolific reactive force field to emerge has been the ReaxFF approach of van Duin and co-workers. ${ }^{86}$ This has been applied to a wide range of materials including organics, metals and oxides. Although complex to parameterise, once achieved it is very powerful since it allows the chemistry of nanostructures to be approximately captured with a cost far below that of full quantum mechanics. Indeed simulations of up to 0.56 billion atoms have been demonstrated with this method (Figure 1). ${ }^{85}$ Examples include the simulation of the transition metal catalysed formation of precursors to carbon nanotubes ${ }^{87}$ and the hydrogenation of boron nitride nanotubes. ${ }^{88}$ 
Before concluding our consideration of different methodologies for determining the potential energy surface that have been applied to nanoparticles and nanostructured materials, it is important to note that there is no single optimal approach for all problems, be it atomistic or otherwise. Indeed the application of what could be called a hierarchy of simulation techniques or multiparadigm modelling is often the appropriate strategy for many problems. ${ }^{89,90}$ Our attention can now turn to examining the simulation of nanoparticle, given a suitable framework for determining the energy as a function of configuration.

One of the key features of a nanoparticle is its morphology. Macroscopically, the thermodynamic equilibrium shape of a crystal can be defined using the Wulff construction in which the normal distance from a surface plane to the centre of the particle is inversely proportional to the surface energy. ${ }^{91}$ In this way, the most stable facets will dominate the morphology. This has been successfully used to predict the morphologies of crystals in many cases. In principle, this can even be done using simple geometric criteria based on the distance between lattice cleavage planes in the underlying crystal structure, ${ }^{92}$ though the use of an accurate surface energy is superior. An alternative to the use of the surface energy is to employ the attachment energy of a growth slice to determine the morphology. ${ }^{93}$ Here the energy of adding a single growth layer to the surface is effectively computed. Use of the attachment energy can be considered to be a quasi-kinetic approach, in that the morphologies predicted by this quantity are often closer to those observed when growth is under kinetic control. ${ }^{94}$ However, it is still strictly speaking a thermodynamic criterion since no rate constants are required.

As highlighted previously, the above macroscopic view of crystalline morphology breaks down at the nanoscale since contributions other than the surface energies become important. Barnard and Zapol have formulated an expression for the free energy of a nanoparticle that allows for surface, edge and corner contributions and applied it to the morphologies of diamond-structured group IV elements (C, Si and $\mathrm{Ge}) .{ }^{95}$ Here the surface, edge and corner energies were determined through DFT calculations, either in the presence or absence of hydrogen termination of dangling bonds. As is often the case, the free energies were approximated by the internal energies, though this could be 
remedied through lattice dynamical calculation of the phonon contributions. An important feature of this work was the inclusion of surface tension to mimic the presence of an environment - something that will be returned to later. This work demonstrates that the morphology of these related materials is predicted to be element-specific and sizedependent (Figure 2), as well as sensitive to surface coverage by hydrogen.

The calculation of the size-dependent thermodynamic morphology has now been applied to a range of semi-ionic materials, including oxides $\left(\mathrm{TiO}_{2},{ }^{96} \mathrm{ZnO},{ }^{97} \mathrm{ZrO}_{2}{ }^{98}\right)$, sulphides $\left(\mathrm{ZnS},{ }^{99} \mathrm{FeS}_{2}{ }^{100}\right)$, selenides $\left(\mathrm{CdSe}^{101}\right)$ and nitrides $(\mathrm{TiN}) .{ }^{102}$ This approach can also account for surface impurities and varying chemical potentials, leading to stoichiometry changes, through appropriate correction of the energy terms. ${ }^{103}$ Since the energetic terms that go into the model are typically obtained from first principles calculations, the nanoparticles are usually considered to be in contact with a gaseous medium. However, if the energies of surfaces in contact with a solvent can be obtained, then the same approach would describe the morphology in some other environment. For example, through the use of force field methods, instead of quantum mechanical techniques, it would be possible to explore the interfacial energy with a solvent through MD simulation. Alternatively, approximate solvated surface energies can be obtained through the use of continuum solvation models, such as the COSMO method. ${ }^{104}$ When modified to enforce the overall neutrality of the induced charges on the solvent accessible surface, as in the COSMIC formulation, ${ }^{105}$ it is then possible to compute solvation energies in 2-D periodic boundary conditions, thereby obtaining morphologies that depend on the dielectric constant of the surrounding medium.

Minimisation of the free energy of a nanoparticle with respect to its shape is one strategy for obtaining morphology. However, this is only appropriate when a system is at thermodynamic equilibrium. Given that nanoparticles are often synthesised by exploiting kinetic control, other simulation approaches can also be valuable. Kinetic Monte Carlo (KMC) is a powerful technique for simulating growth in such cases. ${ }^{106-108}$ Here the state of the system is propagated by stochastically choosing between different activated pathways with a weight that is proportional to the rate constant for that process. Although KMC can be applied "on-the-fly" to a fully atomistic simulation, it is at its most efficient in lattice form when the system can be discretised into a series of states connected by pre- 
tabulated rate constants. For example, the evolution of a crystallite can be represented by a grid of points that are occupied or not by growth units, where the growth fragments can be in solution, added by vapour deposition, or in different states as part of the particle. The probability of different events is then captured by rate constants that can be estimated from transition state theory, determined directly from explicit MD simulations, or taken from experiment, if known. As an example, this approach has been used to simulate the growth of urea crystals from the nanoscale up to micron size, with both water and methanol as the solvent (Figure 3). ${ }^{109}$ For a fixed concentration of solute, the method will ultimately yield the thermodynamically favoured morphology by dissolution and regrowth. However, the results of kinetic control can equally be observed by manipulating the mass transport of solute within the reaction vessel. Recent work has also shown how nanostructured Pt can be optimised using KMC simulation of growth for catalysis applications. ${ }^{110}$

In a similar manner to the Kinetic Monte Carlo approach, the simulation of nanoparticle morphology can be performed using a purely thermodynamic lattice site model within the cluster expansion method. Here the system is represented by an effective spin Hamiltonian, similar to that highlight earlier for studying the magnetism of nanoparticles. In this case the spin state now represents whether a site is occupied by a component or not. The energy is then expanded as a function of the interactions between neighbouring sites in terms of the spin state. This local site-site interaction energy can be parameterised against explicit atomistic calculations and then used to explore a more complex configurational space. While this cluster expansion approach has been extensively for examining phase diagrams of mixing and solid solutions, ${ }^{111-113}$ the application to sodium alanate $\left(\mathrm{NaAlH}_{4}\right)$ nanoparticles, and thereby hydrogen storage, was recently proposed by Mueller and Ceder. ${ }^{114}$ In this way, they were able to not only predict the morphology of different nanoparticle sizes and compositions containing thousands of formula units (see Figure 4), but could also determine the phase diagram for decomposition reactions as a function of particle size.

While both thermodynamic and KMC modelling represent powerful strategies for the study of morphology, both are often strictly valid only for larger nanoparticles due to the underlying assumptions. In the case of free energy minimisation, it is assumed that the 
energies of surfaces, edges and corners are all independent (i.e. there is no coupling between the contributions of each extended defect), which breaks down in the limit of small sizes. In the case of lattice KMC, the tabulation of rate constants often relies on the existence of a small number of well-characterised states, which can also fail to be true at the lower bound of particle size. Hence, at the lower size limit it becomes necessary to switch to a fully atomistic simulation of the entire particle. Fortunately, in this regime it also becomes computationally tractable.

The first challenge when simulating a nanoparticle is often to find the global energy minimum or, for complex structures, an ensemble of low free energy states. As noted above, the morphology may be very different from that of the macroscopic crystal and this is typified by the behaviour of some metals where icosahedral shapes occur for small particles (up to $\sim 4,000$ atoms for some simulations of gold). ${ }^{115}$ Even more extreme are materials such as $\mathrm{ZnO}$ and $\mathrm{ZnS}$, where calculations show that small clusters containing up 94 atoms consist of cage structures more reminiscent of nanoporous materials and that have no bulk analogue for these materials. ${ }^{116}$ For some classes of systems, particularly rare gases, metals, buckminsterfullerene and water, there have been extensive investigations into the cluster minima as a function of size and energetic model. ${ }^{117}$ However, there remains a vast region of uncharted configuration space.

For small clusters, conventional MD may be sufficient to explore the relevant structures. However, as genuinely nanoscale objects are reached the limited timescale currently accessible will prevent all minima from being visited should any of the activation energies be significantly greater than ambient thermal energy. Many techniques now exist to accelerate the exploration of configuration space. In the framework of MD there is replica exchange/parallel tempering ${ }^{118,119}$ for a fixed potential energy surface, while there are also many bias acceleration techniques, including temperature accelerated dynamics ${ }^{120}$ and metadynamics ${ }^{121}$ that seek to assist the escape from potential wells. Metadynamics is particularly advantageous since it can be possible to reconstruct an approximate free energy landscape from the simulation. The choice of a suitable set of collective coordinates to bias can be challenging, though new developments may alleviate this problem. ${ }^{122}$ Beyond MD there are also many other techniques, including Monte Carlo simulated annealing and genetic algorithms. 
Additionally, there are basin/minima-hopping algorithms that can exploit either static or dynamic means of exploring the underlying energy surface. ${ }^{123,124}$ Further information regarding the exploration of the structure of nanostructured systems can be found elsewhere. ${ }^{125,126}$

To date, the majority of theoretical studies of nanoparticles have focused on in vacuo conditions. However, most real applications of such systems involve trapping the nanoparticles in a suitable environment to prevent coalescence. For quantum dots, the alignment of the band gap of the embedding material is essential to achieving quantum confinement. One of the most studied non-metallic systems is silicon nanoparticles, which have been amenable to large-scale electronic structure calculations in both pure and doped forms. ${ }^{127-130}$ Here the most common approach is to saturate dangling bonds with hydrogen, though other molecular passivating groups have also been considered. ${ }^{131}$ Furthermore, it is computationally favourable as it keeps the number of additional electrons/basis functions to a minimum, but also introduces almost no extra complexity in the nuclear configuration space. Given the history of hydrogen termination of dangling bonds within QM/MM schemes, this is a natural approach to take. More challenging is the case of embedding nanoparticles in other solids, as can be used for chalcogenide quantum dots. Here the problem is to find a host material ideally with a commensurate crystal structure and only a small degree of lattice mismatch, before even contemplating the electronic suitability. For silicon nanoparticles, one way of achieving embedding is to use amorphous silica, thereby providing a medium with the flexibility to accommodate extraneous particles. However, care is needed to ensure representative amorphous structures are adopted for use in computational models. ${ }^{132,133}$ For more ionic materials, particles are often simulated in vacuum with no surface modification, or can be embedded in a flexible medium, such as a liquid or a polymer. ${ }^{134}$ When ionic solids are placed in contact with solvents, and water especially, then this opens up the possibility of dissolution and growth occurring. These processes can be driven by either the solution concentration, e.g. by supersaturating the solution with excess ions, or by the different solubilities of distinct surface sites leading to a change in morphology. Here the challenge is that the rate of crystallisation processes is often too slow for explicit atomistic 
simulation. However, the use of supercritical water as a solvent can be used to accelerate nanoparticle growth. ${ }^{135,136}$

One notable phenomenon for nanoparticles is the modification of phase transitions as a function of size. Chen et $a l^{137}$ have demonstrated that the activation barrier to convert four-fold (wurtzite) to six-fold (rock salt) coordination in CdSe and CdS increases with particle size, but with an asymptotic limit that exceeds the bulk value due to the absence of defects. In other cases, the stable polymorph at the nanoscale can differ from that for the bulk material. One of the greatest challenges is to simulate these changes in phase behaviour. Methodologies for simulating bulk phase transitions have become established. For facile cases, the use of the Parrinello-Rahman approach is sufficient, though often this leads to over-heating or pressurisation in order to induce the transition on a timescale currently accessible to MD simulation. The advent of metadynamics using the cell vectors as collective variables has seen several successful explorations of phase transitions for solids. ${ }^{138}$ In the case of nanoparticles, the situation has several key differences. Firstly, a different collective variable must be used since there is no longer a periodic cell. Here local order parameters, such as those due to Steinhardt have proved particularly effective. ${ }^{139}$ Secondly, in order to study a pressure-induced phase transition there needs to be a hydrostatic medium to create the external pressure. Here LennardJones liquids can be used, ${ }^{140}$ though others have employed an ideal gas. ${ }^{141}$ Finally, the simulation of phase transitions in nanoparticles has one advantage over the bulk case, in that it is no longer constrained by the requirement to remain commensurate with a periodic cell, which can biased the outcome of bulk simulations. Arguably the state-ofthe-art in simulating nanoparticle phase transitions is to utilize transition path sampling ${ }^{142}$ to create an ensemble of reactive pathways, as exemplified by studies of CdSe (Figure $5) .{ }^{143}$

A caveat to all studies of phase transitions, such as those above, is to recognize that many theoretical techniques fail to accurately describe phase diagrams. Since a phase transition depends on the crossing of two almost parallel lines, just a small error in the relative energy of two polymorphs can lead to a substantial error. For force field methods particular case must be taken. In the case of II-VI semiconductors, the widely used Tersoff model fails to discriminate between the wurtzite and zinc blende tetrahedral 
structures, giving them as isoenergetic. In contrast, many ionic models for these phases yield a stability difference, but normally with the wrong sign unless an explicit four-body torsional interaction is included. ${ }^{144}$

Beyond the properties of single entities, there are assemblies of nanoparticles, as highlighted during the discussion of mesoscale simulation. Here fewer atomistic simulations have been attempted due to the even greater complexity of trying to probe meaningful minima. One strategy worth noting is that of Sayle and co-workers known as "simulated amorphisation and recrystallisation". ${ }^{145}$ Here a sample of bulk material is annealed at high temperatures within an expanded cell to induce melting, followed by quenching. This induces the formation of polycrystalline materials with inherent defects to give a sample of possible microstructures. By adapting this procedure, thin films or assemblies of nanoparticles can be generated. ${ }^{146,147}$

The above section describes some of the techniques that can be used to simulate materials on the nanoscale. The focus of the next part of the review will be to highlight three case studies, which use some of the above techniques, to fully understand the structure and relative phase stability of ionic and semi-ionic nanoparticles.

\section{Examples of the simulation of nanoparticles}

\section{Titania $\left(\mathrm{TiO}_{2}\right)$}

Titanium dioxide has attracted considered attention as a material for technological applications. Not only is it widely used as a non-toxic white pigment, but also its adsorption properties can be exploited for other purposes. When sensitised by dye molecules, titania can be used for solar cells. ${ }^{148}$ It can also be effective as a photocatalyst and for water splitting. ${ }^{149}$ These qualities have led to diverse uses, including self-cleaning windows and environmental degradation of organic pollutants. By nanoscale engineering it is possible to alter the adsorption properties with the aim of increasing efficiency. Furthermore, there is interest in using lithium intercalation in nanostructured titania (such as in the layer-structured $\mathrm{TiO}_{2}-\mathrm{B}$ polymorph) as a battery material. ${ }^{150}$ 
Despite being extensively studied, bulk $\mathrm{TiO}_{2}$ remains a difficult material to simulate theoretically in some regards. First principles calculations of the relative stability of rutile and anatase often to lead to the latter being the stable phase, ${ }^{151}$ in contradiction to experiment for the bulk limit, as opposed to the finite surface area case. ${ }^{152}$ With some functionals rutile can even possess an imaginary phonon thereby driving a ferroelectric distortion. ${ }^{153}$ There also exists a plethora of force fields from formal charged shell descriptions to variable charge reactive models ${ }^{154}$ with their own pros and cons. ${ }^{155}$

Understanding the factors that control the phase stability, growth, and phase transformation kinetics in nanoparticles is critical to quantify the material behaviour. In this regard titanium dioxide is a particularly interesting material to study because under ambient conditions the rutile phase (space group $\mathrm{P}_{2} / \mathrm{mnm}$ ) is the thermodynamically stable phase relative to the other naturally occurring phases of brookite (space group $P c a b$ ) and anatase (space group $I 4_{1} / a m d$ ). Much of the early investigation into the phase stability as a function of size of the different polymorphs of nanocrystalline titania was performed by Zhang and Banfield. They showed that the thermodynamic stability is particle-size dependent, and at particle diameters below ca. $14 \mathrm{~nm}$, anatase is more stable than rutile. ${ }^{156}$ Zhang and Banfield applied thermodynamic and kinetic analysis to understand the phase transition during growth and aggregation of nanocrystalline $\mathrm{TiO}_{2}$. In their study they concluded that the sequence of transformations between the three polymorphs of anatase, rutile and brookite, is size dependent. Due to the very small differences in energy between the three phases, only a small change in surface energy will alter the stable phase from one polymorph to another. ${ }^{157}$

Naicker et al. took this characterisation of $\mathrm{TiO}_{2}$ polymorphs further by applying MD simulations to describe the structure, heat capacities and surface energies. ${ }^{158}$ Assuming that the nanoparticles are spherical, the authors calculated the surface energy, $U_{\text {surface }}$, according to the expression;

$$
U_{\text {surface }}=\frac{U_{\text {cluster }} n U_{\text {bulk }}}{4 r^{2}}
$$


where $U_{\text {cluster }}$ is the potential energy of the nanoparticle, $U_{\text {bulk }}$ is the potential energy per $\mathrm{TiO}_{2}$ unit in the bulk material, $n$ is the number of $\mathrm{TiO}_{2}$ units in the nanoparticle, and $r$ is the radius of the nanoparticle. This equation is the spherical analogue of the one used to describe the surface energies of two-dimensional surfaces. ${ }^{159}$ The calculated surface energies for a size range of 2-6 nm shows that rutile has a higher surface energy than anatase. Furthermore, Naicker et al. demonstrate that the surface energy contribution is sufficient to alter the order of phase stability with size, especially for anatase, which is found to exhibit the strongest variation.

Aside from those mentioned above, there have been many other molecular dynamics simulations of titania nanoparticles, too numerous to mention all of them. These studies examine various aspects including sintering ${ }^{160,161}$ and amorphous nanoparticles. ${ }^{162,163}$ In addition, there have been first principles calculations of nanoclusters in vacuo. ${ }^{164}$ However, the computational expense of exploring many configurations limits the application of quantum mechanical methods more to the study of relatively ordered systems, such as titania surfaces or nanoribbons. ${ }^{165}$

To give some consideration to the influence of environmental conditions Koparde and Cummings used MD simulations to describe the interactions of rutile and anatase nanoparticles, with a size range of 2.5-4.0 nm, in a box of water. ${ }^{166}$ Here simulations were performed at both ambient conditions and in a hydrothermal environment $(523 \mathrm{~K}$ and $50 \mathrm{kbar}$ ) resembling the regime utilised for industrial processing. Rutile nanoparticles were found to be more hydrophilic than anatase nanoparticles of the same size in terms of the water coverage, with there being two discernable water layers around the surface. No phase transitions were found for the length- and time-scale of their simulations. However, the surfaces of the nanoparticles remained significantly more crystalline when immersed in water, as compared to those in vacuum, due to the water completing the preferred sixfold coordination of titanium. Simulations of approximately spherical rutile particles in water have also been performed in the presence of an ionic atmosphere and static electric fields in order to probe electrophoresis with non-equilibrium molecular dynamics. ${ }^{167}$

It should be noted that the bonding state of water at the surface of $\mathrm{TiO}_{2}$ is an ongoing debate, with it being unclear as to what extent dissociation into $\mathrm{H}^{+}$and $\mathrm{OH}^{-}$may occur, even within first principles studies. ${ }^{168,169}$ When using force field methods it is 
often assumed that dissociation cannot occur due to the nature of most standard water models. Hence, this may influence simulation results. With the use of reactive water models this situation could be alleviated though. ${ }^{170,171}$ A full review of the simulation of titania-water interactions can be found elsewhere. ${ }^{172}$

Barnard et al. applied a thermodynamic model based on the free energy of nanoparticles as a function of size and shape to determine the morphology of anatase and rutile phases with varying surface chemistry. ${ }^{96}$ An important feature of this methodology is to include the weighted surface tensions of the crystallographic surfaces present on the nanoparticle. Therefore to calculate the equilibrium morphology using a Wulff construction Barnard and co-workers use the surface energy and the surface tension applied when an absorbed molecule is present on the surface. By comparing the values of the surface energies and dilation tensor properties of 30 surfaces of rutile and anatase, Barnard et al. found that although the hydrated surfaces result in the lowest surface energies, there is a lack of correlation between stability and the terminations that minimise the surface stress. ${ }^{173}$ This highlights the importance of considering the morphology of a nanoparticle in different chemical environments. In the case of hydrogen-poor and oxygenated surfaces the nanoparticles of both polymorphs become elongated. Anatase nanoparticles are stabilised by surface adsorbates containing a large fraction of hydrogen, whereas rutile nanoparticles are stabilised by larger fractions of oxygen adsorbates. This provides the thermodynamic reasoning behind experimental findings of anatase nanoparticles being grown in acidic conditions. ${ }^{174,175}$ Theoretical calculations have also been used to design the chemical modification of anatase surfaces by fluorine to control the morphology. ${ }^{176}$

There has been considerable interest in minerals on the nanoscale, not only for the case of finite particles, but also nanotubes. Nanotubes of mineral phases are now being extensively studied due to their high surface-to-volume ratio and the curvature of the surface provides increased reactivity compared to nanoparticles of the same phase. One example of this includes the aluminosilicate imogolite, which can exhibit different chiralities similar to carbon nanotubes. ${ }^{177}$ Titanium oxide nanotubes have also been successfully synthesised and studies have indicated that they have improved properties when compared with other forms of titania, especially when applied in photocatalytic ${ }^{178}$ 
and photovoltaic applications. ${ }^{148}$ An excellent review on the application of titanium oxide nanotubes for solar energy production has been given by Mor et al. ${ }^{179}$ Despite their potential applications, the crystal structure of titanium oxide nanotubes remains unclear with several models being proposed, including the trititanate $\left(\mathrm{H}_{2} \mathrm{Ti}_{3} \mathrm{O}_{7}\right),{ }^{180}$ a lepidocrocite-like structure $\left(\mathrm{H}_{\mathrm{x}} \mathrm{Ti}_{2-\mathrm{x} / 4}[]_{\mathrm{x} / 4} \mathrm{O}_{4}\right.$ where $\mathrm{x} \sim 0.7$ and [] is a vacancy)${ }^{181}$ and anatase. ${ }^{182,183}$ This problem has been addressed by Hart et al. who have examined different titania nanotubes constructed by rolling of 2-D sheets to create single-walled materials. They concluded that the most stable structure for titanium oxide nanotubes depends on the activity of water during synthesis. At high water chemical potential there is an increase in hydrogen bonding interactions at the trititanate nanotube surfaces. Therefore nanotubes with diameters greater than $8 \mathrm{~nm}$ appear to be thermodynamically more favourable than bulk trititanate. At low water chemical potential, it appears that tubes generated by chiral wrapping of (101) sheets of anatase are more stable. ${ }^{184}$

The simulations described above provide atomistic detail into the structure and size dependent phase stability of titania nanomaterials. Here the interaction of the surfaces with the surrounding medium clearly plays a vital role in determining the phase stability of nanophase titania. In the next case study we will see how computer simulations can be used to describe the structure and stability of II-VI semiconductors and how we can begin to describe the growth mechanisms of these materials.

\section{Zinc Sulphide}

Binary semiconductors, and in particular chalcogenides, have been extensively studied as examples of quantum dots. During the review of methodologies for nanoparticle simulation we have already touched on materials such as CdSe and CdTe as examples. In this section we briefly review the closely related material zinc sulphide $(\mathrm{ZnS})$, as this has been the focus of much computational scrutiny, leading to some unexpected behaviour for small nanoparticles containing tens of atoms. A full review of this topic can be found elsewhere. ${ }^{185}$ 
When the surfaces of polymorphs of the same material possess different surface energies, a change in phase stability can occur with decreasing particle size. ${ }^{156,186}$ Zinc sulphide has two naturally occurring polymorphs, the more stable cubic sphalerite and the higher energy hexagonal wurtzite form. ${ }^{144}$ These polymorphs are separated by an energy difference that is close to thermal energy at ambient conditions, thereby making it a prime candidate for possible phase changes. Indeed bulk $\mathrm{ZnS}$ exhibits polytypism through the presence of stacking faults, indicating the facile nature of transitioning between polymorphs. Zhang et al. described a ZnS nanoparticle system that under goes structural changes in response to alternations in surface environment rather than particle size. ${ }^{187}$ The average particle diameter determined by UV-vis was $2.8 \mathrm{~nm}$ and $3.0 \mathrm{~nm}$ when determined by transmission electron microscope. This joint experimentalcomputational study showed that the peak position in the XRD spectrum, following the introduction of water to the nanoparticles, went from a disordered structure to a more crystalline sphalerite-like structure. However, interestingly the UV-visible absorption threshold position remained unchanged, indicating no significant size variation. Further evidence from WAXS data showed that the nanocrystalline $\mathrm{ZnS}$ undergoes a room temperature structural modification. The authors conclude that following the binding of water to the surface there is a disorder-to-order transition.

The MD simulations of Zhang et al. show that the interaction between water and $\mathrm{ZnS}$ decreases the interfacial energy, as would be expected. This drives a resultant increase in crystallinity that propagates through the nanoparticle (Figure 6). The simulations of nanocrystalline $\mathrm{ZnS}$ indicate not only that there is a strong interaction between water and the surface, but also that this causes a large stabilisation of the entire nanoparticle. Zhang et al. have also used joint experimental and computational techniques to study the adsorption of other molecules, such as methanol and chlorobenzene, on $\mathrm{ZnS}$ nanoparticles. ${ }^{188}$ The surface structure and core-shell distortion of the nanoparticle are strongly influenced by the environment. The stronger the interaction between the surface and the adsorbed molecule, the more crystalline the structure will be in the nanoparticle.

The above simulations of zinc sulphide focus on particles that are already well and truly at the nanoscale. However, if we are to begin to understand the formation and nucleation of very small clusters of $\mathrm{ZnS}$ we need to be able to confidently predict the 
structure. Although $\mathrm{ZnS}$ nanoparticles have been the subject of a great deal of experimental investigation, little was known regarding the structure and properties of such clusters in the limit of very small sizes. Spano et al. applied a global minimisation technique to investigate the structure of $\mathrm{ZnS}$ clusters in vacuo. ${ }^{116,189}$ To ensure that a global minimum has been found Spano et al. performed at least three different simulated annealing processes for each cluster. The geometries and energies of $\mathrm{ZnS}$ clusters, with sizes ranging from 10 to $47 \mathrm{ZnS}$ units, were simulated using interatomic potentials and then relaxed with first principles techniques to provide more accurate cluster energies. ${ }^{116}$ The simulated annealing process involved a 50 ps molecular dynamics run for every cluster at $3000 \mathrm{~K}$, which were then cooled by $10 \mathrm{~K}$ every 50 ps until a temperature of 300 $\mathrm{K}$ was reached. The high temperature used for the initial steps of the simulated annealing has the effect of removing any possible influence that the initial configuration could have on the final structure. The authors found that the same structures were obtained starting from a random arrangement of atoms or from a cluster cut from the bulk material.

Spano et al. showed that clusters with up to 47 units are more stable with a spheroidal structure than with a bulk like structure. ${ }^{116}$ These "bubble clusters" are formed by the fusing of 4,6 , and 8 atom rings, where all of the atoms are three coordinated. The rings are non-planar distorted polygons; bond distances and bond angles in the same ring are unequal. A strong analogy exists between these cluster structures and the topology of some zeolite cages. For example, the $(\mathrm{ZnS})_{12}$ cluster exhibits a sodalite cage structure as found in faujasite, zeolite-A and sodalite itself. Using DFT techniques they compared the energies of clusters cut from bulk wurtzite and sphalerite with the bubble clusters. The bubble clusters were always more stable due to a reduction of the total dipole across the system. As the cluster size was increased to 60 units of $\mathrm{ZnS}$ Spano et al also found bubble clusters with onion-like structures. ${ }^{189}$

Even larger zinc sulphide clusters have been probed, containing up to 560 formula units. ${ }^{190}$ Here a new structural motif emerges with the particles beginning to adopt something analogous to the BCT framework, rather than wurtzite or sphalerite. However, this observation may in part be an artefact of the force field used, which yields BCT as the most stable bulk phase. Force fields that are designed to target the energetics of $\mathrm{ZnS}$ 
polymorphs ${ }^{144}$ and periodic quantum mechanical calculations are found to give the correct ordering for phase stability. ${ }^{191}$

Using a range of concentrations of zinc and sulphur ions in solution from $0.5 \mathrm{M}$ and 1.25 M Hamad et al. investigated the early stages of nucleation of $\mathrm{ZnS}$ in water. ${ }^{192}$ Initially $\mathrm{Zn}^{2+}$ ions form a stable $\mathrm{Zn}\left(\mathrm{H}_{2} \mathrm{O}\right)_{6}{ }^{2+}$ complex in water. However, these complexes then attract $\mathrm{S}^{2-}$ ions and form ion pairs. They found that there was an activation barrier to forming clusters of $\mathrm{ZnS}$, and only when the concentration of ions was increased did the clusters form readily. Hamad et al. increased the temperature to $500 \mathrm{~K}$ so that more ions would be able to jump the energy barrier and therefore they could study the structures of the clusters that formed. The structures that ultimately formed from solution (Figure 7) were very similar to the bubble structures found in the in vacuo study performed previously. ${ }^{116,189}$ Given the difficulties of simulating crystal growth by direct unbiased molecular dynamics, there are a couple of caveats to this study. Firstly, the concentrations used correspond to a high level of supersaturation and so it remains an open question as to how much this might change the association mechanisms relative to conditions where the system is closer to equilibrium. Secondly, the growth has to be assumed to occur via the $\mathrm{S}^{2-}$ anion in solution because of the use of a non-reactive force field. However, it is unlikely that there is a substantial equilibrium concentration of this species present, except at very high $\mathrm{pH}$, with $\mathrm{SH}^{-}$being the dominant anion. Whether the point of proton transfer and any potential net charge on the clusters would alter the details of growth can only be speculated on at present.

The above investigation of zinc sulphide association in solution highlights some of the challenges faced when trying to use computational techniques to simulate the early stages of growth of clusters and nanoparticles from solution. Ultimately it is often necessary to introduce bias acceleration or to methodically determine free energy profiles for the key reactions to be able to make progress. This will be highlighted in the next example of calcium carbonate, a mineral that has a particular complex formation mechanism.

\section{Calcium carbonate}


The evolution of mineral nanoparticles has a significant impact on the natural world. Nowhere is this perhaps more evident than for the case of calcium carbonate $\left(\mathrm{CaCO}_{3}\right)$. Organisms are able to selectively control the production of this mineral through biomineralisation to create anything from skeletal materials for protection through to optical lenses. This has led to interest in the creation of advanced materials via biomimetic processes, inspired by biomineralization. ${ }^{193}$

While much progress has already been made toward a fundamental understanding of such biomineralization phenomena, especially through the use of atomic force microscopy to study peptide-surface interactions, ${ }^{194-196}$ there remain many important questions regarding the precise mechanism. ${ }^{197}$ Calcium carbonate can occur naturally as three crystalline polymorphs (calcite, aragonite and the disordered vaterite) as well as an amorphous form (ACC) and two hydrates (monohydrocalcite and ikaite). Therefore to fully understand the biological control that is exerted over the selection of polymorphs it is critical to understand the growth of calcium carbonate in aqueous solution, including at the nanoscale.

Typically the growth of minerals is explained via classical nucleation theory. Here ions aggregate to form clusters that are initially unstable due to the entropic penalty for organization and the positive surface free energy of forming the crystal-solvent interface. As the particles increase in size, the cost of the surface energy is outweighed by the bulk lattice energy and so the system passes through a free energy maximum before increasing in stability with growing diameter. ${ }^{198}$ Recent experimental studies ${ }^{199}$ have proven that calcium carbonate grows by a non-classical mechanism in which stable prenucleation clusters occur prior to the formation of amorphous calcium carbonate. Both estimates of the hydrodynamic radius ${ }^{199}$ and the results of cryo-TEM ${ }^{200}$ indicate that some of the initially formed particles have dimensions of a few nanometres. Despite this major advance it remains difficult to unambiguously determine the structure and composition of prenucleation clusters, or the mechanism of their transformation to ACC, from experimental alone. Consequently there is a valuable role for atomistic simulation to play in probing nanostructured calcium carbonate. Although bulk phases of calcium carbonate are readily amenable to first principles quantum mechanics, the study of nanoparticles 
and solution growth is currently largely the realm of force field methods due to the demands of the time- and length-scale required.

To the best of our knowledge, the first published simulations of what might be considered calcium carbonate nanoparticles are due to Bearchell and Heyes. ${ }^{201}$ They simulated clusters with ten formula units of $\mathrm{CaCO}_{3}$ in the presence of surfactant molecules to provide insights relevant to the lubricant industry. Initial particle structures were cleaved from bulk calcite with an approximately spherical morphology. It was observed that the clusters effectively became amorphous during molecular dynamics. This may be a consequence of the starting configuration, since anything other than a rhombohedral morphology composed of the single dominant cleavage plane may be unstable at this dimension. Kerisit et al. also studied the behaviour of small calcite nanoparticles, containing $18 \mathrm{CaCO}_{3}$ units, during a molecular dynamics simulation in vacuo and similarly found that the particles rapidly lost long-range order. However, when the same particle was simulated surrounded by an explicit model of water, the underlying crystal structure of the nanoparticles was preserved for throughout the duration of the simulation. $^{202}$

Cooke and Elliott also used MD simulations to investigate the size dependant stability of calcite nanoparticles and the role of water on stabilising the nanoparticle. ${ }^{197}$ Using the Wulff construction to generate the equilibrium shape, Cooke and Elliott created ten different calcite nanoparticles ranging in size from 18 to $324 \mathrm{CaCO}_{3}$ formula units and with a diameter up to $2.5 \mathrm{~nm}$. To gain molecular level insight into the stability of the nanoparticles as a function of size, they quantified the extent to which the carbonate group rotates during the simulation by defining an order parameter. At a temperature of $300 \mathrm{~K}$ there was little orientational ordering of the carbonates in the three smallest nanoparticles. As the nanoparticles increased in size the orientational ordering of the carbonates also increased. This was attributed to the increased ratio of the number of bulk atoms with surface atoms. Instability of the structure of the nanoparticles was ascribed to under coordination of the atoms on the surface. By considering the radial distribution function $(\mathrm{RDF})$ of the nanoparticles they also found translational disorder of the atoms, which increased as the nanoparticle size decreased. When the nanoparticles were immersed in a box of water the orientational ordering of the carbonates increased with 
respect to the same sized nanoparticle in vacuum. The solvent molecules complete the coordination shell of the surface cations, thereby stabilising the particle. Hence this reduces the need for the surface carbonate groups to rotate, resulting in high values of the order parameter for all the nanoparticles considered. This further illustrates the importance of including a full description of the solvent when describing the structure and stability of nanoparticles as a function of size.

A further complication in the study of nanoparticles of calcium carbonate is that the thermodynamically preferred state may well be the amorphous form, rather than something approaching a crystalline structure. The challenge is therefore to quantify the difference in stability between phases as a function of particle size under conditions where spontaneous transformations will not occur on the timescale of several tens of nanoseconds readily accessible to molecular dynamics. To overcome this problem, Quigley and Rodger used metadynamics to explore the possible structural arrangements of a $2 \mathrm{~nm}$ diameter calcium carbonate nanoparticle. ${ }^{139}$ Here the nanoparticle was constructed in a different manner to previous simulations by melting a bulk aragonite supercell at $2500 \mathrm{~K}$. By removing the periodic boundary conditions, the melted calcium carbonate formed a liquid drop, which was quenched to form an approximately spherical particle of amorphous material. This amorphous nanoparticle, which contained 75 $\mathrm{CaCO}_{3}$, units was then embedded in a box of water. The same size of calcium carbonate particle had previously been shown by Cooke and Elliott to retain order over a nanosecond simulation in water and limited order over $20 \mathrm{~ns}$ simulation in vacuo. ${ }^{197}$ Therefore it could be expected that metadynamics simulations might locate at least partially ordered configurations in addition to amorphous states. During the simulations, indeed examples of both amorphous (Figure 8A) and partly ordered (Figure 8B) particle configurations were explored due to the metadynamics bias, though the free energy basin associated with the amorphous state dominates the energetic landscape. Although not presented in detail, Quigley and Rodger comment that the amorphous state became increasingly dominant with decreasing cluster size.

In a later study, Quigley et al. presented a revision to their methodology so that they could allow simulations to be conducted at constant pressure (rather than constant density) and for larger nanoparticles. ${ }^{203}$ The results of this study concluded that by 
changing the volume available to the nanoparticle and solvent it is possible to manipulate the stable phase, which may have implications for how nature controls its selectivity for a given polymorph.

While being a powerful demonstration of the use of metadynamics to map out the free energy landscape in complex environments, there is an important caveat to note for the above and many other simulations of calcium carbonate, namely that the underlying force field fails to give the proper relative thermodynamics of the different phases. Many of the force fields that have been used for the simulation of this mineral either give aragonite as the stable phase instead of calcite, contrary to experiment, or overstabilise calcite by up to an order of magnitude. Equally as important, most models also fail to describe the free energies of solvation of the ions accurately leading to a solubility product that is in error by tens of orders of magnitude, which can have consequences for the way that clusters interact with the solvating water. Raiteri et al. have recently developed both rigid and flexible interatomic potentials that correctly describe the thermodynamic behaviour of the calcium carbonate-water interface, as well as the correct free energy difference between calcite and aragonite. ${ }^{198,204}$ The second model with a flexible carbonate group also benefits from an improved description of the relative energetics of vaterite.

Using the above revised force fields, Raiteri and Gale have also sort to examine the thermodynamic stability of calcium carbonate nanoparticles. The approach taken to create nanoparticles was similar to previous studies. To create crystalline nanoparticles of calcite they assumed that the most stable basal (10 14) surface plane dominates the morphology. The hexagonal crystal structure of calcite leads to morphologies where all terminations are of this surface resulting in rhombohedral crystallites. To create nanoparticles of amorphous calcium carbonate they followed a similar approach to Quigley and Rodger by annealing particles in vacuo at 3,000 K until all order is removed. Raiteri and Gale took a number of sizes of both crystalline and amorphous nanoparticles (18-864 $\mathrm{CaCO}_{3}$ formula units) and immersed them in water. A graphical representation of the two $864 \mathrm{CaCO}_{3}$ nanoparticles is shown in Figure 9.

Amorphous calcium carbonate is known to actually contain a variable amount of structural water, with an approximate stoichiometry of one to one in some materials. ${ }^{205}$ 
To address this, Raiteri and Gale included a range of water contents within their amorphous nanoparticles. Although the direct determination of relative free energies is complex for such systems, they were able to correct the calculated enthalpy differences with an estimate of the entropy of water incorporation based on experimental data. In this way, it was found that the amorphous nanoparticles exhibit an increasing preference for water incorporation as they grow. Furthermore, the amorphous clusters, with their favoured water content, are lower in free energy than calcite nanoparticles up to a size of $\sim 4 \mathrm{~nm}$, beyond which they become metastable.

In order to begin to probe the thermodynamics of growth, the free energy profile for the addition of ion pairs to different nanoparticles was also determined by umbrella sampling in the above study. The addition of ion pairs to the $(10 \overline{14})$ calcite surface is only favourable energetically when solvent separated due to the ordered layer of water that is present at the interface. In contrast, the free energy profile for addition of ion pairs to amorphous calcium carbonate exhibits almost no activation barrier and leads to a stable bound state. This difference is caused by the amorphous surface frustrating the formation of an ordered interface with the surrounding water. Remarkably, this effect and the resulting free energy profile are almost independent of nanoparticle size or water content. These results support the experimental findings of Gebauer et al. ${ }^{199}$ who proposed a speciation model in which the energetics of ion pair addition is constant for a given coordination number. Furthermore, it is consistent with the non-classical nature of the growth of amorphous calcium carbonate, though the question of how the experimentally observed nucleation occurs remains open.

\section{Conclusions}

In this review we have attempted to capture the current status of atomistic simulation of nanomaterials, with a primary focus on partially ionic nanoparticles, while also trying to place this in the context of other computational approaches. Because of the rapidly expanding nature of the field it is only possible to survey a small fraction of the full literature, and so the main objective has been to highlight the challenges involved in 
predicting structural and morphological properties of semi-ionic nanoparticles. Here the key challenge is to be able to explore a complex potential energy surface sufficiently to identify the important configurations. This is made all the harder by the fact that force field models, and even some density functionals, fail to describe the correct relative energetics of different phases.

Arguably the two greatest challenges for the simulation of nanomaterials going forward are realism and making the connection with experiment. Increasing there are many simulations that make predictions ahead of experiment for nanomaterials in exotic structures. It is therefore the responsibility of simulators to try to ensure that such predictions stay within the bounds of what might be achievable. Optimisation of structures only finds the nearest local minimum, and so serious consideration must always be given to competing forms that might be more stable. If something is metastable, then a strategy for trapping the proposed state should be given. Many calculations are performed on nanoparticles in vacuo, but for technological purposes it is necessary to consider how the surface might be passivated to preserve the cluster in a terrestrial environment. Having designed a realistic pathway for synthesising a given nanoparticle, the second challenge is to use theory to provide guidance on how the prediction might be experimentally validated. Increasingly calculations are becoming able to make useful predictions of spectroscopic properties, such as NMR and optical spectra. Therefore it is feasible to assist in the detection of a predicted nanomaterial in cases where direct structural interpretation of the experiments may be difficult.

In all the case studies highlighted above it can be seen that to fully understand the behaviour of nanoparticles and their relative phase stability there needs to be an accurate description of the surrounding medium. As the power of computers increases, more and more research is moving away from in vacuo studies and the level of complexity is increasing. There are already several examples in the literature of single mineral nanoparticles in boxes of explicit water. ${ }^{206-208}$ In the limit of larger nanoparticles predictions can be made of size dependent morphology based on the adsorption of water vapour. However, for aqueous environments it is ultimately necessary to move beyond monolayer coverage of water and to consider the interface with bulk liquid. For many minerals and ionic materials, speciation then becomes an issue and so it becomes 
necessary to use either quantum mechanical methods or a reactive force field capable of describing dissociation of water. Ultimately multiscale techniques may well be required to handle the dual requirements of large system sizes and chemical reactivity in particular regions. Furthermore, the current trend toward massively parallel computing will also demand a re-examination of some of the underlying algorithms. For example, instead of plane wave density functional theory being the de facto standard for first principles calculation, real space systematic algorithms involving multi-resolution basis sets may well become the norm for calculations on heterogeneous nanomaterials. ${ }^{209,210}$

When a nanoparticle is immersed in a complex environment, such as a liquid or polymer, then the process of determining energetics becomes more complex. Instead of computing just enthalpies, the consideration of free energies becomes mandatory as the impact of a nanoparticle on the entropy of the surrounding medium can no longer be regarded as negligible. As shown above, free energy calculations, such as umbrella sampling and metadynamics have been successfully used to describe the structure and growth of preformed amorphous calcium carbonate. Future computer simulations should include free energy calculations to study other processes, such as the aggregation of nanoparticles in solution, in cases where mesoscale models are inappropriate. One example of where atomistic simulations could be used to explain a natural phenomenon is oriented attachment. Oriented attachment involves spontaneous self-organisation of adjacent particles so that they share a common crystallographic orientation. ${ }^{211}$ It occurs readily in nature and has been observed in a range of different materials. ${ }^{211-214}$ Atomistic simulations could be used to explain how this process occurs and predict the conditions that favour this type of crystal growth. The ability to direct crystallisation through oriented attachment is an exciting prospect that could allow for the creation of new nanostructures with well-defined sizes and shape. ${ }^{215}$ The majority of computer simulations on the aggregation of nanoparticles have been performed in vacuum to date, ${ }^{215-218}$ and so a challenge for the future is to include the surrounding medium.

As discussed in the above case studies, one approach to describe oriented attachment could be free energy calculations. However, to utilise free energy approaches more widely can be non-trivial though since it is necessary to have a well-defined reaction coordination or a small number of collective variables to be explored. Even where a good 
choice is made there can be problems if other degrees of freedom are not explored rapidly enough. This is highlighted by the case of agglomeration, where the distance between the nanoparticles is sampled as a reaction coordinate. In the presence of a medium, the rotation of nanoparticles will be slow and so there is no guarantee a unique free energy profile will be obtained in a finite sampling time. Despite this, there is good cause for optimism as rapid progress is being made in the development of collective variables to accelerate the timescale for exploring configuration space. ${ }^{122}$

Atomistic computer simulation of nanomaterials has already shown itself to be a valuable complement to experimental observation. As the complexity of nanoscience and nanotechnology increases, so will the opportunities for modelling, making for an exciting future in this area of research.

\section{Acknowledgements}

The authors thank the Australian Research Council for funding under the Discovery Program, as well as Kate Wright, Paolo Raiteri and Zoe Taylor for useful discussions. 


\section{Figure Captions}

Figure 1: Reactive simulation of a $20 \mathrm{~nm}$ aluminium nanoparticle with oxygen gas. Snapshot is taken after $40 \mathrm{ps}$ and already shows the partial formation of a non-stoichiometric aluminium oxide layer at the surface. Sphere size of small and large denotes aluminium and oxygen, respectively, while the colour coding indicates the temperature of the atoms. ${ }^{85}$ Reprinted with permission from P. Vashishta, R. K. Kalia and A. Nakano, Journal of Physical Chemistry B, 2006, 110, 3727-3733. Copyright 2011 American Chemical Society.

Figure 2: Morphologies of pure/hydrogen terminated carbon (as diamond) (a/d), silicon (b/e), and germanium (c/f) nanoparticles as a function of size (increasing from left to right) as computed by Barnard and Zapol. ${ }^{95}$ Reprinted with permission from A. S. Barnard and P. Zapol, Journal of Chemical Physics, 2004, 121, 4276-4283. Copyright 2011, American Institute of Physics.

Figure 3: Crystallite morphologies for urea grown from water (left) and methanol (right) obtained from kinetic Monte Carlo simulation and optical microscopy. Particles start at the nanoscale and can evolve to the micron-scale during the time of the simulation. ${ }^{109}$ Reprinted by permission from Macmillan Publishers Ltd: S. Piana, M. Reyhani and J. D. Gale, Nature, 2005, 438, 70-73, copyright 2011.

Figure 4: Illustration of the application of the cluster expansion method to nanoparticle morphology for the case of sodium alanate decomposition. Predicted ground-state morphology for nanoparticles consisting of (a) 15000 atoms of $\mathrm{NaAlH}_{4}$, (b) 5000 atoms of $\mathrm{NaH}$, (c) 9000 atoms of $\mathrm{Na}_{3} \mathrm{AlH}_{6}$, (d) 2500 atom Al. ${ }^{114}$ Reprinted with permission from T. Mueller and G. Ceder, ACS Nano, 2010, 4, 5647-5656. Copyright 2011 American Chemical Society.

Figure 5: Simulation of the nucleation of rock salt (blue) structured CdSe within a hexagonal (grey) nanoparticle of size $\sim 50 \AA{ }^{143}$ In (a) the critical nucleus is illustrated as a function of pressure while in (b) a series of time slices are shown to indicate the shearing of (100) planes after 13 ps. Reprinted with permission from M. Grunwald and C. Dellago, Nano Letters, 2009, 9, 2099-2102. Copyright 2011 American Chemical Society.

Figure 6: Snapshots taken from the molecular dynamics simulation of an approximately $3 \mathrm{~nm}$ nanoparticle of $\mathrm{ZnS}$, with zinc and oxygen coloured yellow and red, respectively. The upper images (a) indicate the nanoparticle viewed from different directions after simulation in vacuo, while those in (b) represent the same system after simulation in water. ${ }^{187}$ Reprinted by permission from Macmillan Publishers Ltd: H. Zhang, B. Gilbert, F. Huang and J. Banfield, Nature, 2003, 424, 1025-1029, copyright 2011. 
Figure 7: Formation of clusters of $\mathrm{ZnS}$ ( $\mathrm{Zn}$ are blue and $\mathrm{S}$ are yellow in colour) at $500 \mathrm{~K}$ from a supersaturated solution. ${ }^{192}$ Reprinted with permission from S. Hamad, S. Cristol and C.R.A. Catlow, J. Am. Chem. Soc., 2005, 127, 2580-2590. Copyright 2011 American Chemical Society.

Figure 8: Sample configurations illustrating both amorphous (A) and ordered (B) nanoparticles of calcium carbonate in water during metadynamics simulation of a 75 formula unit nanoparticle. ${ }^{139}$ Reprinted with permission from D. Quigley and P. M. Rodger, Journal of Chemical Physics, 2008, 128, 221101. Copyright 2011, American Institute of Physics.

Figure 9: Illustration of two 864 formula unit nanoparticles of (a) calcite and (b) anhydrous amorphous calcium carbonate when immersed in water. The van der Waals surface of the nanoparticle has been shown in grey, with part of it removed to allow the inner structure to become visible. ${ }^{198}$ Reprinted with permission from P. Raiteri and J. D. Gale, J Am Chem Soc, 2010, 132, 17623-17634. Copyright 2011 American Chemical Society. 


\section{References}

(1) M. Gratzel, Journal of Photochemistry and Photobiology C-Photochemistry Reviews, 2003, 4, 145-153.

(2) Y. Cui and C. M. Lieber, Science, 2001, 291, 851-853.

(3) S. Mornet, S. Vasseur, F. Grasset and E. Duguet, Journal of Materials Chemistry, 2004, 14, 2161-2175.

(4) L. B. Hu, J. W. Choi, Y. Yang, S. Jeong, F. La Mantia, L. F. Cui and Y. Cui, Proceedings of the National Academy of Sciences of the United States of America, 2009, 106, 21490-21494.

(5) D. Astruc, F. Lu and J. R. Aranzaes, Angewandte Chemie-International Edition, 2005, 44, 78527872 .

(6) M. C. Roco, C. A. Mirkin and M. C. Hersam Nanotechnology Research Directions for Societal Needs in 2020; Springer: Berlin and Boston, 2010.

(7) D. K. Kim, M. Mikhaylova, Y. Zhang and M. Muhammed, Chemistry of Materials, 2003, 15, 1617-1627.

(8) S. Van Aert, K. J. Batenburg, M. D. Rossell, R. Erni and G. Van Tendeloo, Nature, 2011, 470, 374-377.

(9) Z. Y. Li, N. P. Young, M. Di Vece, S. Palomba, R. E. Palmer, A. L. Bleloch, B. C. Curley, R. L. Johnston, J. Jiang and J. Yuan, Nature, 2008, 451, 46-U2.

(10) C. A. Ohlin, E. M. Villa, J. R. Rustad and W. H. Casey, Nature Materials, 2010, 9, 11-19.

(11) M. Terrones, Annual Review of Materials Research, 2003, 33, 419-501.

(12) F. Banhart, N. Grobert, M. Terrones, J. C. Charlier and P. M. Ajayan, International Journal of Modern Physics B, 2001, 15, 4037-4069.

(13) P. M. Ajayan, Chemical Reviews, 1999, 99, 1787-1799.

(14) A. Sanchez, S. Abbet, U. Heiz, W. Schneider, H. Hakkinen, R. Barnett and U. Landman, J Phys Chem A, 1999, 103, 9573-9578.

(15) J. F. Parker, C. A. Fields-Zinna and R. W. Murray, Accounts of Chemical Research, 2010, 43, 1289-1296.

(16) L. Xiao, L. Zhuang, Y. Liu, J. T. Lu and H. D. Abruna, Journal of the American Chemical Society, 2009, 131, 602-608.

(17) P. W. Tasker, Journal of Physics C-Solid State Physics, 1979, 12, 4977-4984.

(18) N. Metropolis and S. Ulam, Journal of the American Statistical Association, 1949, 44, 335-341.

(19) B. J. Alder and T. E. Wainwright, Journal of Chemical Physics, 1959, 31, 459-466.

(20) J. M. Luttinger and W. Kohn, Physical Review, 1955, 97, 869-883.

(21) A. Zunger, Mrs Bulletin, 1998, 23, 35-42.

(22) K. Yang and Y. Q. Ma, Nature Nanotechnology, 2010, 5, 579-583.

(23) H. Noguchi and M. Takasu, Biophysical Journal, 2002, 83, 299-308.

(24) A. Y. Sinyagin, A. Belov, Z. N. Tang and N. A. Kotov, Journal of Physical Chemistry B, 2006, 110, 7500-7507.

(25) Z. L. Zhang, Z. Y. Tang, N. A. Kotov and S. C. Glotzer, Nano Letters, 2007, 7, 1670-1675.

(26) E. M. Purcell and Pennypac.Cr, Astrophysical Journal, 1973, 186, 705-714.

(27) T. R. Jensen, G. C. Schatz and R. P. Van Duyne, Journal of Physical Chemistry B, 1999, 103, 2394-2401.

(28) E. Hao, G. C. Schatz and J. T. Hupp, Journal of Fluorescence, 2004, 14, 331-341.

(29) H. Kachkachi, A. Ezzir, M. Nogues and E. Tronc, European Physical Journal B, 2000, 14, 681-

689.

(30) R. H. Kodama and A. E. Berkowitz, Physical Review B, 1999, 59, 6321-6336.

(31) R. H. Kodama, A. E. Berkowitz, E. J. Mcniff and S. Foner, Physical Review Letters, 1996, 77, 394-397.

(32) F. H. Streitz and J. W. Mintmire, Journal of Adhesion Science and Technology, 1994, 8, 853-864.

(33) P. Jemmer, M. Wilson, P. A. Madden and P. W. Fowler, Journal of Chemical Physics, 1999, 111, 2038-2049. 
(34) S. M. Woodley, P. D. Battle, C. R. A. Catlow and J. D. Gale, Journal of Physical Chemistry B, 2001, 105, 6824-6830.

(35) A. Warshel and R. M. Weiss, Journal of the American Chemical Society, 1980, 102, 6218-6226.

(36) R. Armiento and A. Mattsson, Phys. Rev. B, 2005, 72, 085108.

(37) Z. Wu and R. Cohen, Phys. Rev. B, 2006, 73, 235116.

(38) J. P. Perdew, A. Ruzsinszky, G. I. Csonka, O. A. Vydrov, G. E. Scuseria, L. A. Constantin, X. Zhou and K. Burke, Phys. Rev. Lett., 2008, 100, 136406.

(39) A. D. Becke, Journal of Chemical Physics, 1993, 98, 1372-1377.

(40) J. Heyd and G. E. Scuseria, Journal of Chemical Physics, 2004, 121, 1187-1192.

(41) S. Grimme, Journal of Computational Chemistry, 2004, 25, 1463-1473.

(42) S. Grimme, Journal of Computational Chemistry, 2006, 27, 1787-1799.

(43) M. Dion, H. Rydberg, E. Schroder, D. C. Langreth and B. I. Lundqvist, Physical Review Letters, 2004, 92.

(44) G. Roman-Perez and J. M. Soler, Physical Review Letters, 2009, 103.

(45) J. P. Perdew and A. Zunger, Physical Review B, 1981, 23, 5048-5079.

(46) E. Runge and E. K. U. Gross, Physical Review Letters, 1984, 52, 997-1000.

(47) K. Yabana and G. F. Bertsch, Physical Review B, 1996, 54, 4484-4487.

(48) A. Tsolakidis, D. Sanchez-Portal and R. M. Martin, Physical Review B, 2002, 66.

(49) Y. K. Lei, H. J. Liu and W. Xiao, Modelling and Simulation in Materials Science and Engineering, 2010, 18.

(50) S. A. Shevlin and S. M. Woodley, Journal of Physical Chemistry C, 2010, 114, 17333-17343.

(51) S. Auvinen, M. Alatalo, H. Haario, J. P. Jalava and R. J. Lamminmaki, Journal of Physical Chemistry C, 2011, 115, 8484-8493.

(52) F. De Angelis, Chemical Physics Letters, 2010, 493, 323-327.

(53) M. Pastore and F. De Angelis, Acs Nano, 2010, 4, 556-562.

(54) F. De Angelis, A. Tilocca and A. Selloni, Journal of the American Chemical Society, 2004, 126, 15024-15025.

(55) R. Sanchez-De-Armas, J. Oviedo, M. A. S. Miguel and J. F. Sanz, Journal of Physical Chemistry C, 2011, 115, 11293-11301.

(56) A. N. Enyashin and G. Seifert, Physical Chemistry Chemical Physics, 2007, 9, 5772-5775.

(57) A. N. Enyashin and A. L. Ivanovskii, Physica E-Low-Dimensional Systems \& Nanostructures, 2005, 30, 164-168.

(58) B. Goswami, S. Pal and P. Sarkar, Journal of Physical Chemistry C, 2008, 112, 11630-11636.

(59) J. Robles, O. Mayorga, T. S. Lee and D. Diaz, Nanostructured Materials, 1999, 11, 283-286.

(60) Y. Nosaka and H. Tanaka, Journal of Physical Chemistry B, 2002, 106, 3389-3393.

(61) J. Frenzel, J. O. Joswig and G. Seifert, Journal of Physical Chemistry C, 2007, 111, 10761-10770.

(62) J. M. Soler, E. Artacho, J. D. Gale, A. Garcia, J. Junquera, P. Ordejon and D. Sanchez-Portal, Journal of Physics-Condensed Matter, 2002, 14, 2745-2779.

(63) J. N. Kim, F. Mauri and G. Galli, Physical Review B, 1995, 52, 1640-1648.

(64) A. M. N. Niklasson, Physical Review B, 2002, 66.

(65) W. T. Yang and T. S. Lee, Journal of Chemical Physics, 1995, 103, 5674-5678.

(66) M. S. Daw and M. I. Baskes, Physical Review Letters, 1983, 50, 1285-1288.

(67) M. I. Baskes, Physical Review B, 1992, 46, 2727-2742.

(68) J. Tersoff, Physical Review Letters, 1988, 61, 2879-2882.

(69) N. A. Marks, Physical Review B, 2001, 63.

(70) D. W. Brenner, O. A. Shenderova, J. A. Harrison, S. J. Stuart, B. Ni and S. B. Sinnott, Journal of Physics-Condensed Matter, 2002, 14, 783-802.

(71) J. H. Los, L. M. Ghiringhelli, E. J. Meijer and A. Fasolino, Physical Review B, 2005, 72.

(72) G. C. Abell, Physical Review B, 1985, 31, 6184-6196.

(73) S. J. Stuart, A. B. Tutein and J. A. Harrison, Journal of Chemical Physics, 2000, 112, 6472-6486.

(74) M. J. Sanders, M. Leslie and C. R. A. Catlow, Journal of the Chemical Society-Chemical Communications, 1984, 1271-1273.

(75) J. Applequist, Journal of Chemical Physics, 1985, 83, 809-826.

(76) A. W. Dick and B. G. Overhauser, Physical Reviews, 1958, 112, 90-103. 
(77) J. D. Gale and K. Wright In Theoretical and Computational Methods in Mineral Physics: Geophysical Applications 2010; Vol. 71, p 391-411.

(78) T. S. Mahadevan and S. H. Garofalini, Journal of Physical Chemistry B, 2007, 111, 8919-8927.

(79) F. H. Streitz and J. W. Mintmire, Physical Review B, 1994, 50, 11996-12003.

(80) T. Iwasaki and H. Miura, Journal of Materials Research, 2001, 16, 1789-1794.

(81) X. W. Zhou, H. N. G. Wadley, J. S. Filhol and M. N. Neurock, Physical Review B, 2004, 69.

(82) S. Alavi, J. W. Mintmire and D. L. Thompson, Journal of Physical Chemistry B, 2005, 109, 209-

214.

(83) T.-R. Shan, B. D. Devine, J. M. Hawkins, A. Asthagiri, S. R. Phillpot and S. B. Sinnott, Physical Review B, 2010, 82.

(84) T.-R. Shan, B. D. Devine, J. M. Hawkins, A. Asthagiri, S. R. Phillpot and S. B. Sinnott, Physical Review $B, 2010,82$.

(85) P. Vashishta, R. K. Kalia and A. Nakano, Journal of Physical Chemistry B, 2006, 110, 3727 3733.

(86) A. C. T. Van Duin, S. Dasgupta, F. Lorant and W. A. Goddard, Journal of Physical Chemistry A, 2001, 105, 9396-9409.

(87) K. D. Nielson, A. C. T. Van Duin, J. Oxgaard, W. Q. Deng and W. A. Goddard, Journal of Physical Chemistry A, 2005, 109, 493-499.

(88) S. S. Han, J. K. Kang, H. M. Lee, A. C. T. Van Duin and W. A. Goddard, Journal of Chemical Physics, 2005, 123, 114703-114708.

(89) J. Q. Broughton, F. F. Abraham, N. Bernstein and E. Kaxiras, Physical Review B, 1999, 60, 2391-2403.

(90) M. J. Buehler, A. C. T. Van Duin and W. A. Goddard, Physical Review Letters, 2006, 96, 4.

(91) G. Wulff, Z Krystallogr Minera, 1901, 34, 449-530.

(92) J. D. H. Donny and D. Harker, American Mineralogist, 1937, 22, 446-467.

(93) P. Hartman and P. Bennema, Journal of Crystal Growth, 1980, 49, 145-156.

(94) P. M. Oliver, G. W. Watson, E. T. Kelsey and S. C. Parker, Journal of Materials Chemistry, 1997, 7, 563-568.

(95) A. S. Barnard and P. Zapol, Journal of Chemical Physics, 2004, 121, 4276-4283.

(96) A. S. Barnard, P. Zapol and L. A. Curtiss, Journal of Chemical Theory and Computation, 2005, 1, 107-116.

(97) A. S. Barnard, Y. Xiao and Z. Cai, Chemical Physics Letters, 2006, 419, 313-316.

(98) A. S. Barnard, R. R. Yeredla and H. F. Xu, Nanotechnology, 2006, 17, 3039-3047.

(99) C. Feigl, S. P. Russo and A. S. Barnard, Journal of Materials Chemistry, 2010, 20, 4971-4980.

(100) A. S. Barnard and S. P. Russo, J. Phys. Chem. C, 2007, 111, 11742-11746.

(101) A. S. Barnard, H. Xu, X. Li, N. Pradhan and X. Peng, Nanotechnology, 2006, 17, 5707-5714.

(102) A. S. Barnard, Journal of Computational and Theoretical Nanoscience, 2004, 1, 334-339.

(103) A. S. Barnard and S. P. Russo, J. Mater. Chem., 2009, 19, 3389-3394.

(104) A. Klamt and G. Schuurmann, Journal of the Chemical Society-Perkin Transactions 2, 1993, 799-805.

(105) J. D. Gale and A. L. Rohl, Molecular Simulation, 2007, 33, 1237-1246.

(106) C. C. Battaile, D. J. Srolovitz and J. E. Butler, Journal of Crystal Growth, 1998, 194, 353-368.

(107) A. Violi and A. Venkatnathan, Journal of Chemical Physics, 2006, 125.

(108) T. H. Lim, D. Mccarthy, S. C. Hendy, K. J. Stevens, S. A. Brown and R. D. Tilley, Acs Nano, 2009, 3, 3809-3813.

(109) S. Piana, M. Reyhani and J. D. Gale, Nature, 2005, 438, 70-73.

(110) V. Gorshkov, O. Zavalov, P. B. Atanassov and V. Privman, Langmuir, 2011, 27, 8554-8561.

(111) J. W. D. Connolly and A. R. Williams, Physical Review B, 1983, 27, 5169-5172.

(112) M. T. Dove, S. Thayaparam, V. Heine and K. D. Hammonds, American Mineralogist, 1996, 81, 349-362.

(113) V. L. Vinograd, M. H. F. Sluiter, B. Winkler, A. Putnis, U. Halenius, J. D. Gale and U. Becker, Mineralogical Magazine, 2004, 68, 101-121.

(114) T. Mueller and G. Ceder, Acs Nano, 2010, 4, 5647-5656.

(115) Y. G. Chushak and L. S. Bartell, Journal of Physical Chemistry B, 2001, 105, 11605-11614.

(116) E. Spano, S. Hamad and C. Catlow, J Phys Chem B, 2003, 107, 10337-10340. 
(117) J. P. K. Doye, D. J. Wales, W. Branz and F. Calvo, Physical Review B, 2001, 64.

(118) Y. Sugita and Y. Okamoto, Chemical Physics Letters, 1999, 314, 141-151.

(119) U. H. E. Hansmann, Chemical Physics Letters, 1997, 281, 140-150.

(120) M. R. Sorensen and A. F. Voter, Journal of Chemical Physics, 2000, 112, 9599-9606.

(121) A. Laio and M. Parrinello, Proceedings of the National Academy of Sciences of the United States of America, 2002, 99, 12562-12566.

(122) G. A. Tribello, M. Ceriotti and M. Parrinello, Proceedings of the National Academy of Sciences of the United States of America, 2010, 107, 17509-17514.

(123) D. J. Wales and J. P. K. Doye, Journal of Physical Chemistry A, 1997, 101, 5111-5116.

(124) S. Goedecker, Journal of Chemical Physics, 2004, 120, 9911-9917.

(125) C. R. A. Catlow, S. T. Bromley, S. Hamad, M. Mora-Fonz, A. A. Sokol and S. M. Woodley, Phys. Chem. Chem. Phys., 2010, 12, 786-811.

(126) D. C. Sayle and R. L. Johnston, Current Opinion in Solid State \& Materials Science, 2003, 7, 312.

(127) B. Delley and E. F. Steigmeier, Physical Review B, 1993, 47, 1397-1400.

(128) S. Ogut, J. R. Chelikowsky and S. G. Louie, Physical Review Letters, 1997, 79, 1770-1773.

(129) M. Rohlfing and S. G. Louie, Physical Review Letters, 1998, 80, 3320-3323.

(130) J. R. Chelikowsky, M. M. G. Alemany, T. L. Chan and G. M. Dalpian, Reports on Progress in Physics, 2011, 74.

(131) Z. Y. Zhou, L. Brus and R. Friesner, Nano Letters, 2003, 3, 163-167.

(132) G. Hadjisavvas and P. C. Kelires, Physical Review Letters, 2004, 93.

(133) P. Kroll and H. J. Schulte, Physica Status Solidi B-Basic Solid State Physics, 2006, 243, R47-R49.

(134) H. Kasemagi, M. Klintenberg, A. Aabloo and J. O. Thomas, Solid State Ionics, 2002, 147, $367-$

375.

(135) I. G. Nahtigal, A. Y. Zasetsky and I. M. Svishchev, Journal of Physical Chemistry B, 2008, 112, 7537-7543.

(136) N. Lummen and B. Kvamme, Physical Chemistry Chemical Physics, 2009, 11, 9504-9513.

(137) C. C. Chen, A. B. Herhold, C. S. Johnson and A. P. Alivisatos, Science, 1997, 276, 398-401.

(138) R. Martonak, A. Laio and M. Parrinello, Physical Review Letters, 2003, 90.

(139) D. Quigley and P. M. Rodger, Journal of Chemical Physics, 2008, 128, 221101.

(140) B. J. Morgan and P. A. Madden, Nano Letters, 2004, 4, 1581-1585.

(141) M. Grunwald and C. Dellago, Molecular Physics, 2006, 104, 3709-3715.

(142) C. Dellago, P. G. Bolhuis, F. S. Csajka and D. Chandler, Journal of Chemical Physics, 1998, 108, 1964-1977.

(143) M. Grunwald and C. Dellago, Nano Letters, 2009, 9, 2099-2102.

(144) K. Wright and J. D. Gale, Physical Review B, 2004, 70.

(145) D. C. Sayle and G. W. Watson, Journal of Physical Chemistry B, 2001, 105, 5506-5514.

(146) X. D. Feng, D. C. Sayle, Z. L. Wang, M. S. Paras, B. Santora, A. C. Sutorik, T. X. T. Sayle, Y. Yang, Y. Ding, X. D. Wang and Y. S. Her, Science, 2006, 312, 1504-1508.

(147) D. C. Sayle and G. W. Watson, Journal of Physical Chemistry B, 2002, 106, 10793-10807.

(148) S. Uchida, R. Chiba, M. Tomiha, N. Masaki and M. Shirai, Electrochemistry, 2002, 70, 418-420.

(149) M. Ni, M. K. H. Leung, D. Y. C. Leung and K. Sumathy, Renewable \& Sustainable Energy Reviews, 2007, 11, 401-425.

(150) L. Kavan, M. Kalbac, M. Zukalova, I. Exnar, V. Lorenzen, R. Nesper and M. Graetzel, Chemistry of Materials, 2004, 16, 477-485.

(151) J. Muscat, V. Swamy and N. M. Harrison, Physical Review B, 2002, 65.

(152) A. Navrotsky, Proceedings of the National Academy of Sciences of the United States of America, 2004, 101, 12096-12101.

(153) B. Montanari and N. M. Harrison, Chemical Physics Letters, 2002, 364, 528-534.

(154) V. Swamy and J. D. Gale, Physical Review B, 2000, 62, 5406-5412.

(155) B. S. Thomas, N. A. Marks and B. D. Begg, Physical Review B, 2004, 69.

(156) H. Z. Zhang and J. F. Banfield, Journal of Materials Chemistry, 1998, 8, 2073-2076.

(157) H. Z. Zhang and J. F. Banfield, Journal of Physical Chemistry B, 2000, 104, 3481-3487.

(158) P. K. Naicker, P. T. Cummings, H. Z. Zhang and J. F. Banfield, Journal of Physical Chemistry B, 2005, 109, 15243-15249. 
(159) G. W. Watson, E. T. Kelsey, N. H. De Leeuw, D. J. Harris and S. C. Parker, J Chem Soc Faraday T, 1996, 92, 433-438.

(160) V. N. Koparde and P. T. Cummings, Journal of Physical Chemistry B, 2005, 109, 24280-24287.

(161) V. N. Koparde and P. T. Cummings, Acs Nano, 2008, 2, 1620-1624.

(162) V. Van Hoang, Journal of Physics D-Applied Physics, 2007, 40, 7454-7461.

(163) H. Z. Zhang, B. Chen, J. F. Banfield and G. A. Waychunas, Physical Review B, 2008, 78.

(164) M. H. Weng, C. Chen and S. P. Ju, Chinese Journal of Catalysis, 2009, 30, 384-390.

(165) T. He, F. C. Pan, Z. X. Xi, X. J. Zhang, H. Y. Zhang, Z. H. Wang, M. W. Zhao, S. S. Yan and Y. Y. Xia, Journal of Physical Chemistry C, 2010, 114, 9234-9238.

(166) V. N. Koparde and P. T. Cummings, J. Phys. Chem. C, 2007, 111, 6920-6926.

(167) N. J. English and W. F. Long, Physica a-Statistical Mechanics and Its Applications, 2009, 388, 4091-4096.

(168) N. Kumar, S. Neogi, P. R. C. Kent, A. V. Bandura, J. D. Kubicki, D. J. Wesolowski, D. Cole and J. O. Sofo, Journal of Physical Chemistry C, 2009, 113, 13732-13740.

(169) L. M. Liu, C. J. Zhang, G. Thornton and A. Michaelides, Physical Review B, 2010, 82.

(170) J. W. Halley, J. R. Rustad and A. Rahman, Journal of Chemical Physics, 1993, 98, 4110-4119.

(171) U. W. Schmitt and G. A. Voth, Journal of Physical Chemistry B, 1998, 102, 5547-5551.

(172) C. H. Sun, L. M. Liu, A. Selloni, G. Q. Lu and S. C. Smith, Journal of Materials Chemistry, 2010, 20, 10319-10334.

(173) A. S. Barnard, P. Zapol and L. A. Curtiss, Surface Science, 2005, 582, 173-188.

(174) T. Sugimoto, X. P. Zhou and A. Muramatsu, Journal of Colloid and Interface Science, 2003, 259,

43-52.

(175) T. Sugimoto, X. P. Zhou and A. Muramatsu, Journal of Colloid and Interface Science, 2003, 259,

53-61.

(176) H. G. Yang, C. H. Sun, S. Z. Qiao, J. Zou, G. Liu, S. C. Smith, H. M. Cheng and G. Q. Lu, Nature, 2008, 453, 638-U4.

(177) R. Demichelis, Y. Noel, P. D'arco, L. Maschio, R. Orlando and R. Dovesi, Journal of Materials Chemistry, 2010, 20, 10417-10425.

(178) M. Adachi, Y. Murata, M. Harada and S. Yoshikawa, Chemistry Letters, 2000, 942-943.

(179) G. K. Mor, O. K. Varghese, M. Paulose, K. Shankar and C. A. Grimes, Solar Energy Materials and Solar Cells, 2006, 90, 2011-2075.

(180) Q. Chen, G. H. Du, S. Zhang and L. M. Peng, Acta Crystallographica Section B-Structural Science, 2002, 58, 587-593.

(181) R. Z. Ma, Y. Bando and T. Sasaki, Chemical Physics Letters, 2003, 380, 577-582.

(182) T. Kasuga, M. Hiramatsu, A. Hoson, T. Sekino and K. Niihara, Langmuir, 1998, 14, 3160-3163.

(183) W. Z. Wang, O. K. Varghese, M. Paulose, C. A. Grimes, Q. L. Wang and E. C. Dickey, Journal of Materials Research, 2004, 19, 417-422.

(184) J. N. Hart, S. C. Parker and A. A. Lapkin, Acs Nano, 2009, 3, 3401-3412.

(185) S. Hamad, S. M. Woodley and C. R. A. Catlow, Molecular Simulation, 2009, 35, 1015-1032.

(186) J. M. Mchale, A. Auroux, A. J. Perrotta and A. Navrotsky, Science, 1997, 277, 788-791.

(187) H. Zhang, B. Gilbert, F. Huang and J. Banfield, Nature, 2003, 424, 1025-1029.

(188) H. Zhang, B. Chen, Y. Ren, G. A. Waychunas and J. F. Banfield, Phys. Rev. B, 2010, 81, 125444.

(189) E. Spano, S. Hamad and C. Catlow, Chem. Commun., 2004, 864-865.

(190) S. Hamad and C. R. A. Catlow, Journal of Crystal Growth, 2006, 294, 2-8.

(191) Z. Taylor, P. Raiteri and J. D. Gale, (in preparation).

(192) S. Hamad, S. Cristol and C. Catlow, J. Am. Chem. Soc., 2005, 127, 2580-2590.

(193) S. Mann Biomineralization: Principles and Concepts in Bio-inorganic Materials Oxford UniverSity Press: Oxford, 2001.

(194) L. Addadi and S. Weiner, Proceedings of the National Academy of Sciences of the United States of America, 1985, 82, 4110-4114.

(195) A. Wierzbicki, C. S. Sikes, J. D. Madura and B. Drake, Calcified Tissue International, 1994, 54, 133-141.

(196) J. J. De Yoreo In Perspectives on Inorganic, Organic, and Biological Crystal Growth: From Fundamentals to Applications 2007; Vol. 916, p 416-438.

(197) D. J. Cooke and J. A. Elliott, J Chem Phys, 2007, 127, 104706. 
(198) P. Raiteri and J. D. Gale, J Am Chem Soc, 2010, 132, 17623-17634.

(199) D. Gebauer, A. Volkel and H. Colfen, Science, 2008, 322, 1819-1822.

(200) E. M. Pouget, P. H. H. Bomans, J. Goos, P. M. Frederik, G. De With and N. Sommerdijk, Science, 2009, 323, 1455-1458.

(201) C. A. Bearchell and D. M. Heyes, Molecular Simulation, 2002, 28, 517-538.

(202) S. Kerisit, D. J. Cooke, D. Spagnoli and S. C. Parker, J. Mater. Chem., 2005, 15, 1454-1462.

(203) D. Quigley, C. L. Freeman, J. H. Harding and P. M. Rodger, J Chem Phys, 2011, 134, 044703.

(204) P. Raiteri, J. D. Gale, D. Quigley and P. M. Rodger, J. Phys. Chem. C, 2010, 114, 5997-6010.

(205) F. M. Michel, J. Macdonald, J. Feng, B. L. Phillips, L. Ehm, C. Tarabrella, J. B. Parise and R. J. Reeder, Chemistry of Materials, 2008, 20, 4720-4728.

(206) G. A. Tribello, F. Bruneval, C. Liew and M. Parrinello, J Phys Chem B, 2009, 113, 11680-11687.

(207) D. Spagnoli, B. Gilbert, G. A. Waychunas and J. F. Banfield, Geochimica et Cosmochimica Acta, 2009, 73, 4023-4033.

(208) D. Spagnoli, J. P. Allen and S. C. Parker, Langmuir, 2011, 27, 1821-1829.

(209) R. J. Harrison, G. I. Fann, T. Yanai, Z. Gan and G. Beylkin, Journal of Chemical Physics, 2004, 121, 11587-11598.

(210) L. Genovese, A. Neelov, S. Goedecker, T. Deutsch, S. A. Ghasemi, A. Willand, D. Caliste, O. Zilberberg, M. Rayson, A. Bergman and R. Schneider, Journal of Chemical Physics, 2008, 129.

(211) R. Penn and J. Banfield, Science, 1998, 281, 969-971.

(212) J. Banfield and H. Zhang, Rev Mineral Geochem, 2001, 44, 1-58.

(213) J. Banfield, S. Welch, H. Zhang, T. Ebert and R. Penn, Science, 2000, 289, 751-754.

(214) R. Penn and J. Banfield, American Mineralogist, 1998, 83, 1077-1082.

(215) M. Alimohammadi and K. A. Fichthorn, Nano Letters, 2009, 9, 4198-4203.

(216) H. Zhang and J. Banfield, Nano Lett, 2004, 4, 713-718.

(217) D. Spagnoli, J. F. Banfield and S. C. Parker, J. Phys. Chem. C, 2008, 112, 14731-14736.

(218) P. Martin, D. Spagnoli, A. Marmier, S. C. Parker, D. C. Sayle and G. Watson, Molecular Simulation, 2006, 32, 1079-1093. 


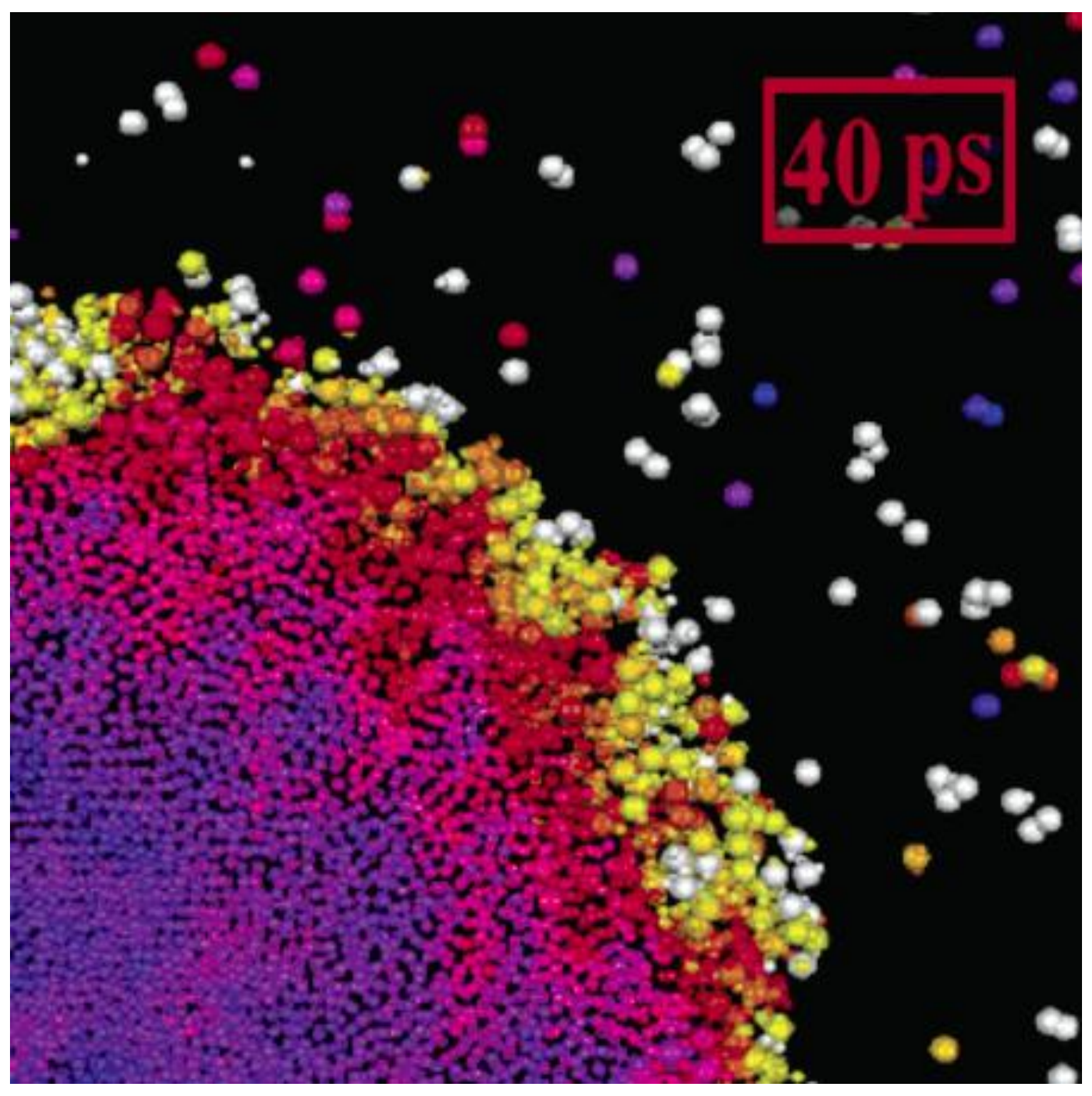

Figure 1 

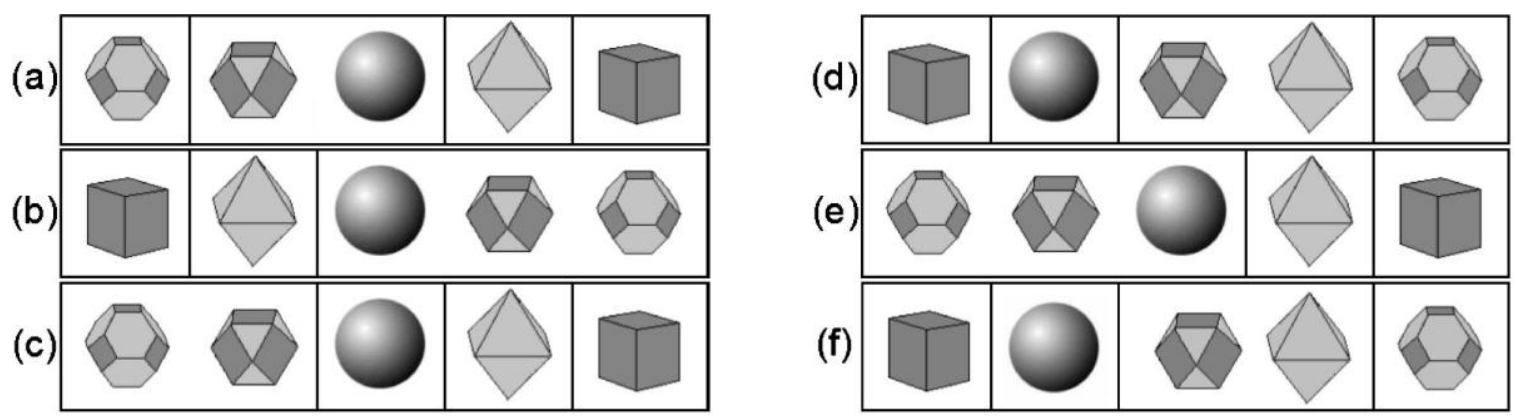

Figure 2 


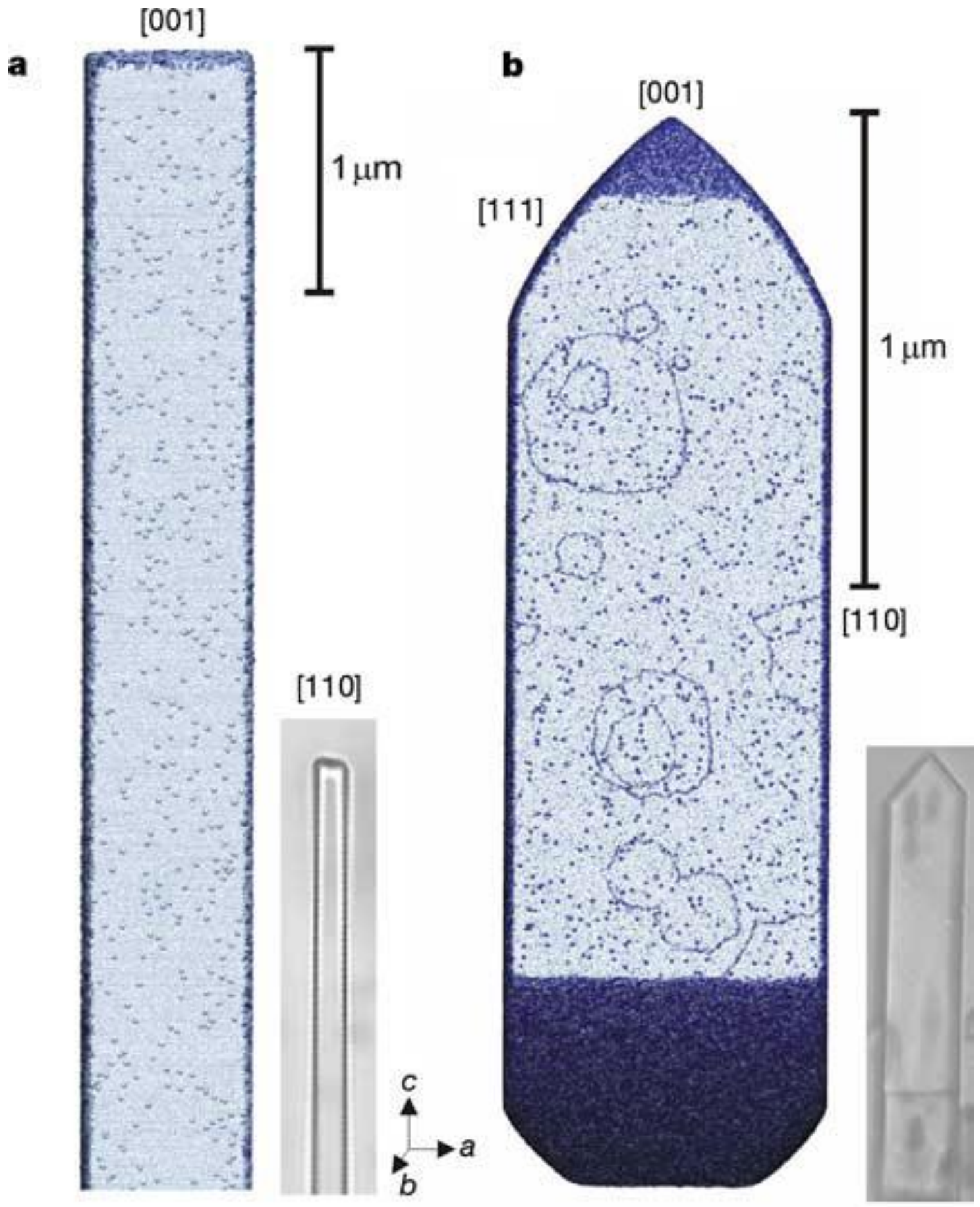

Figure 3 

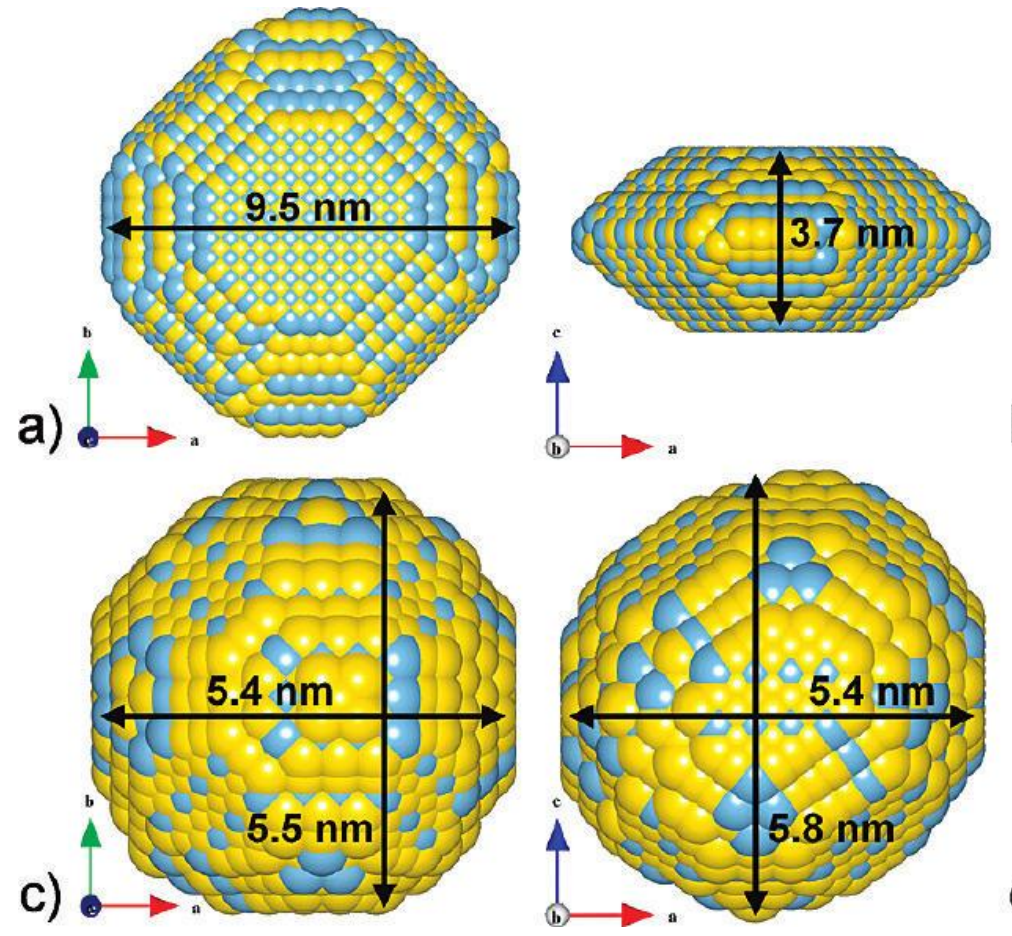

b)
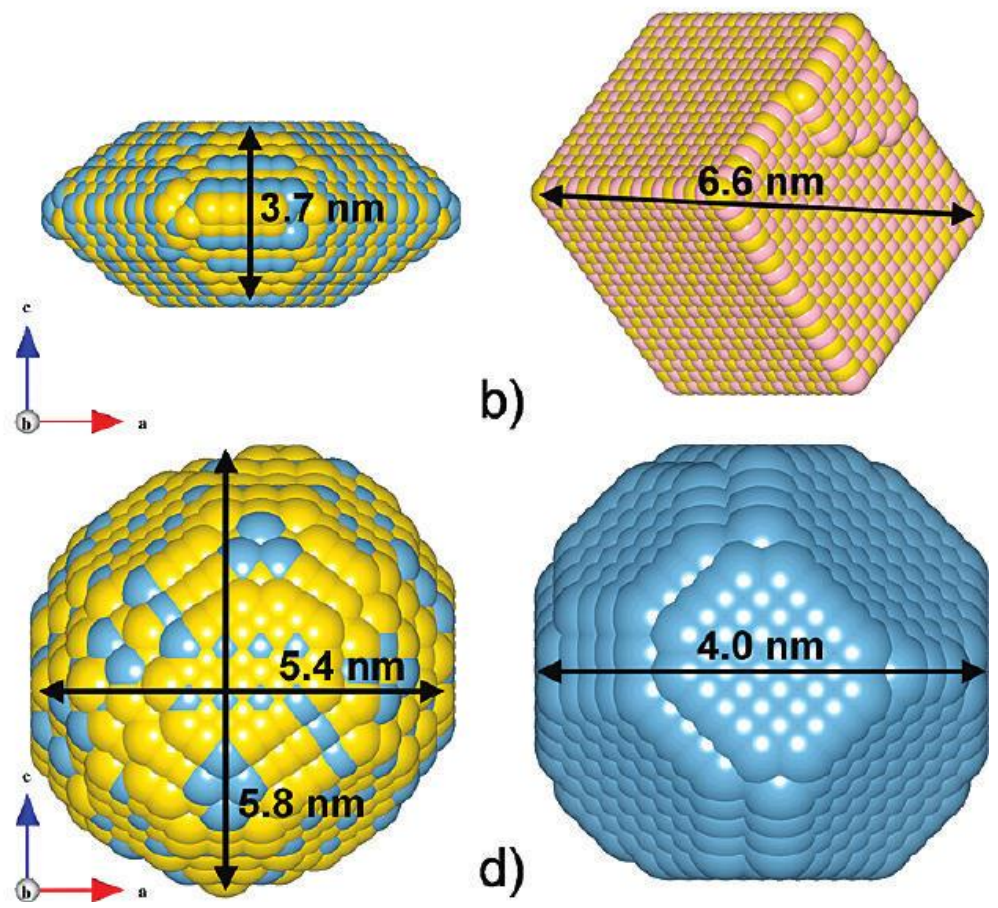

Figure 4 
(a)
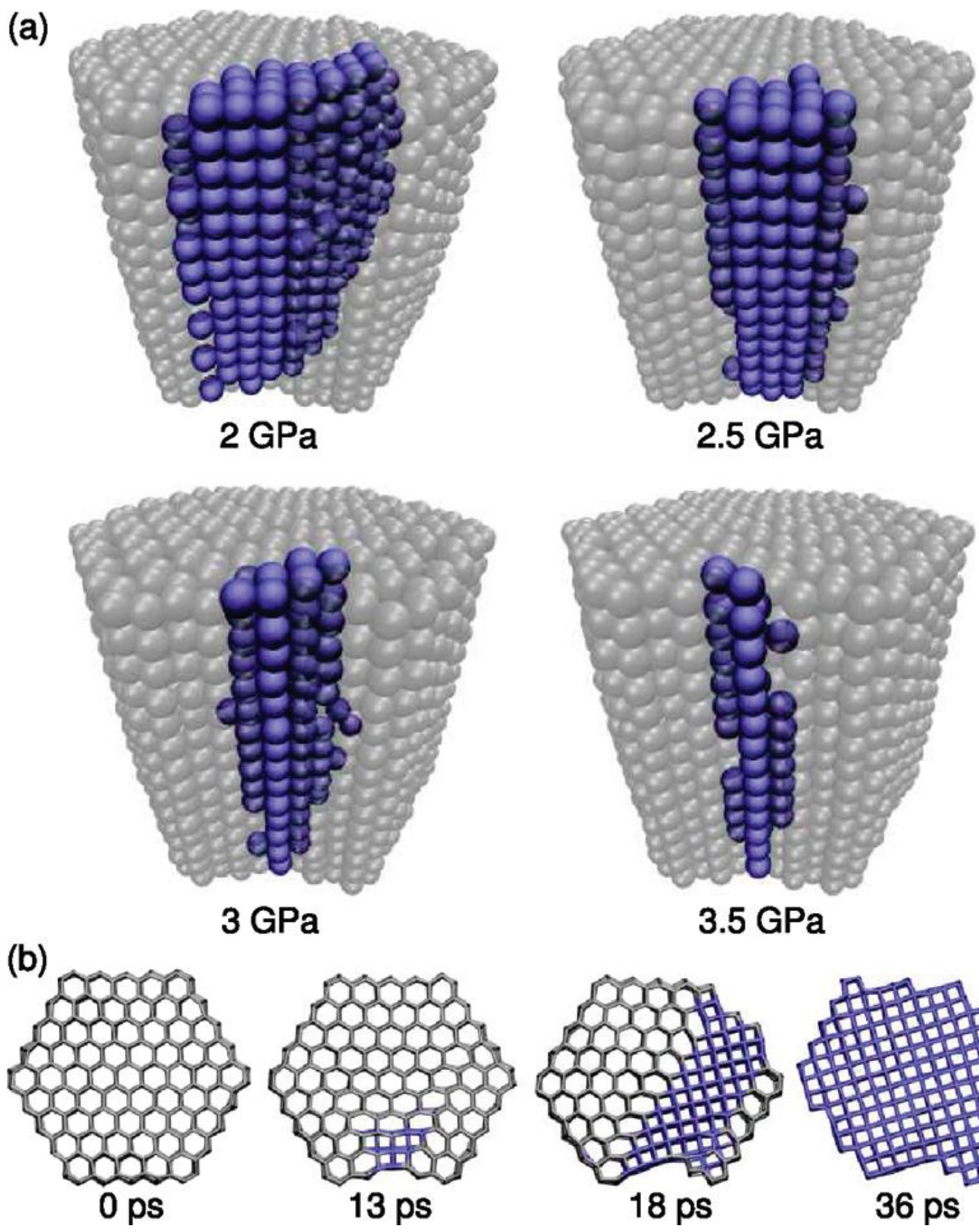

Figure 5
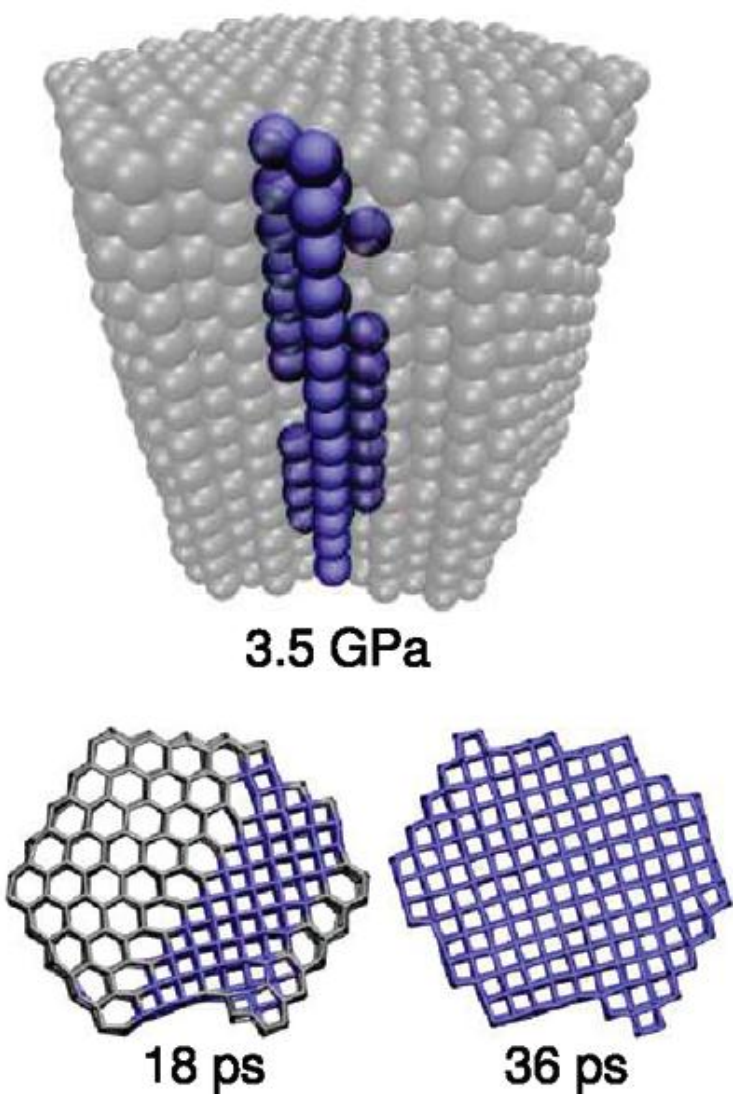

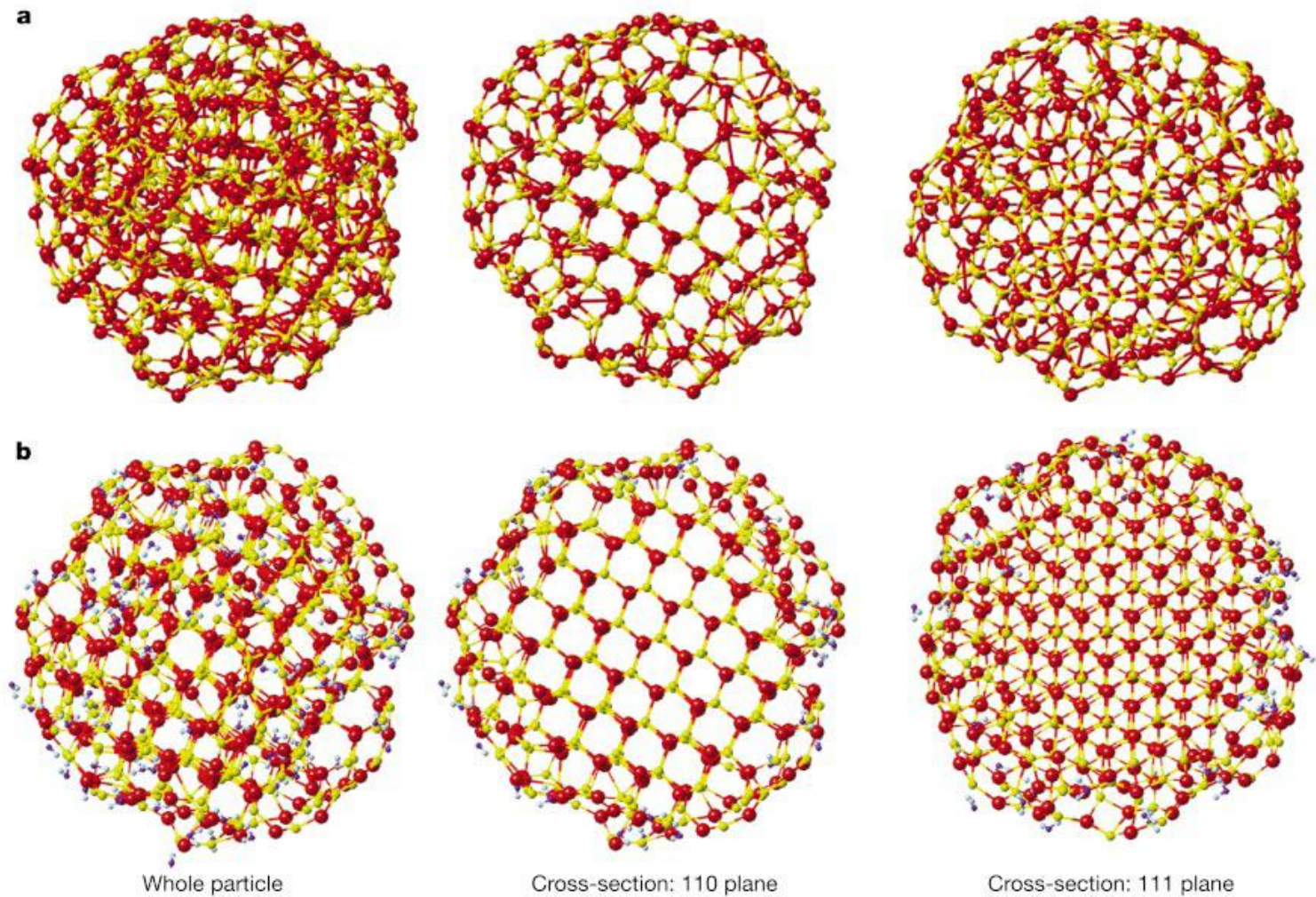

Figure 6 

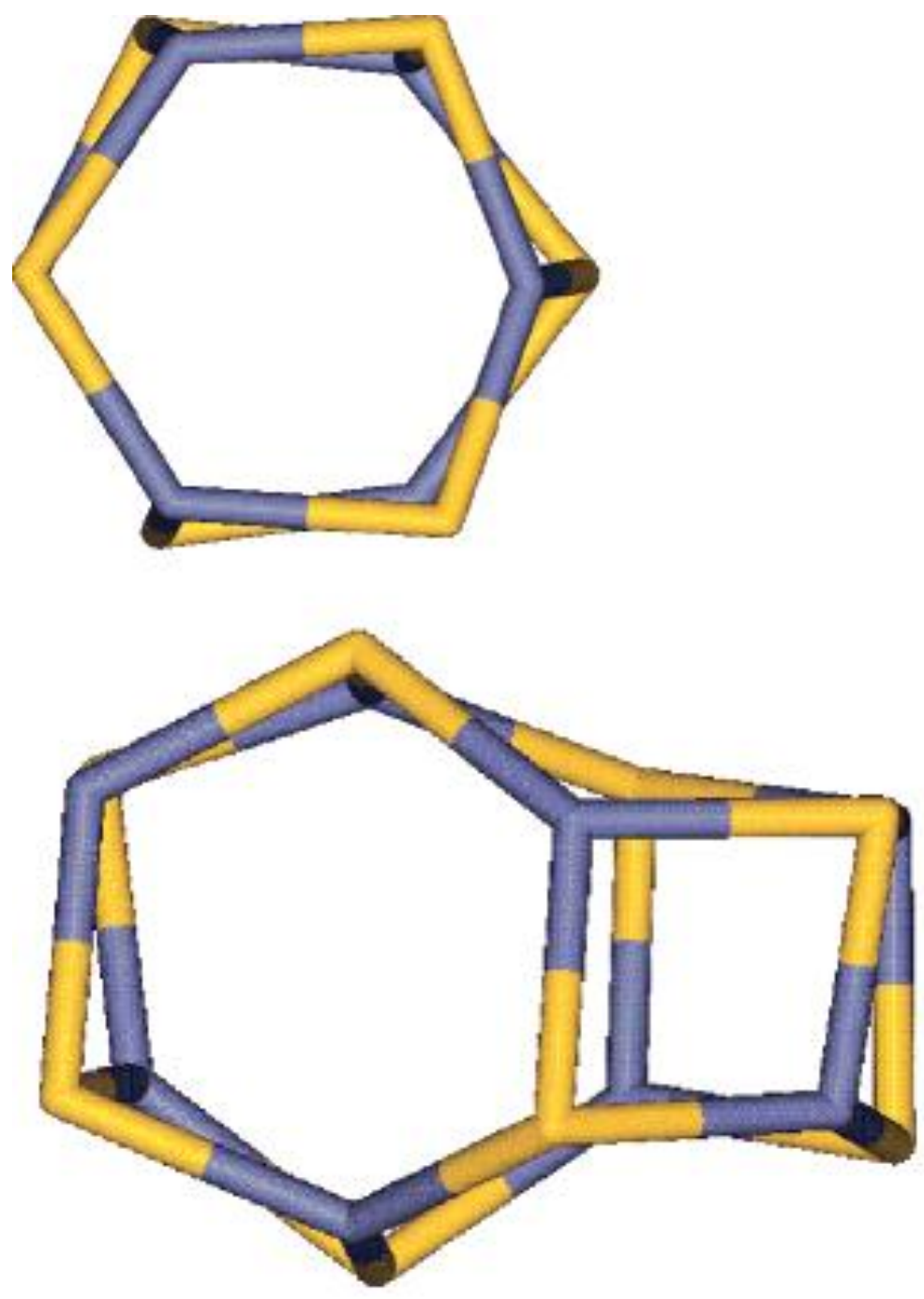

Figure 7

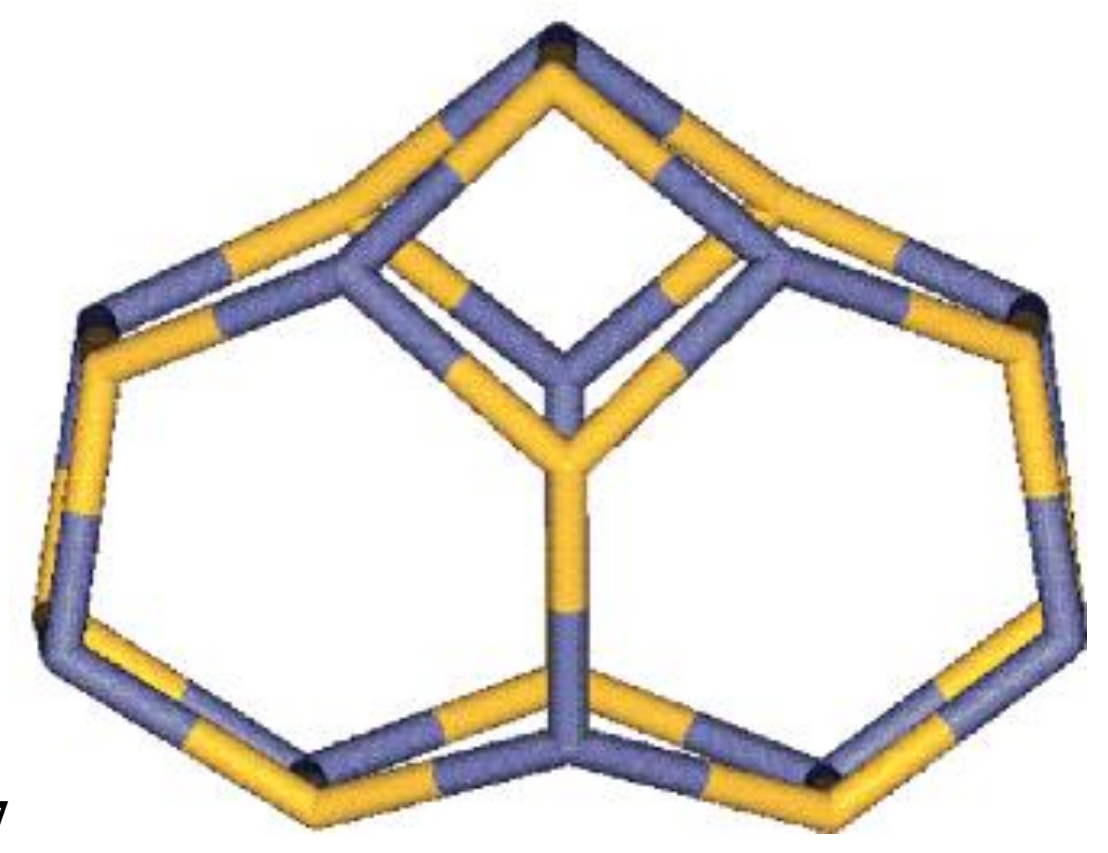




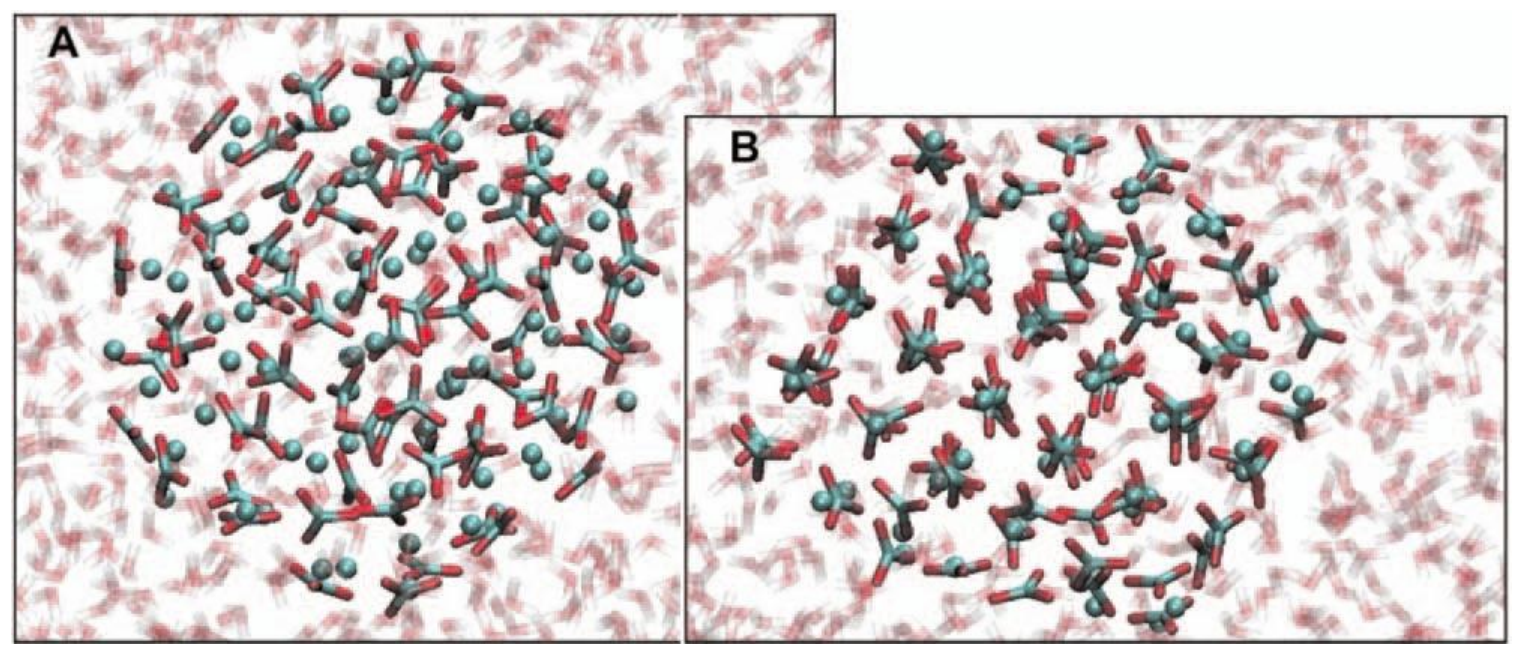

Figure 8 
a)

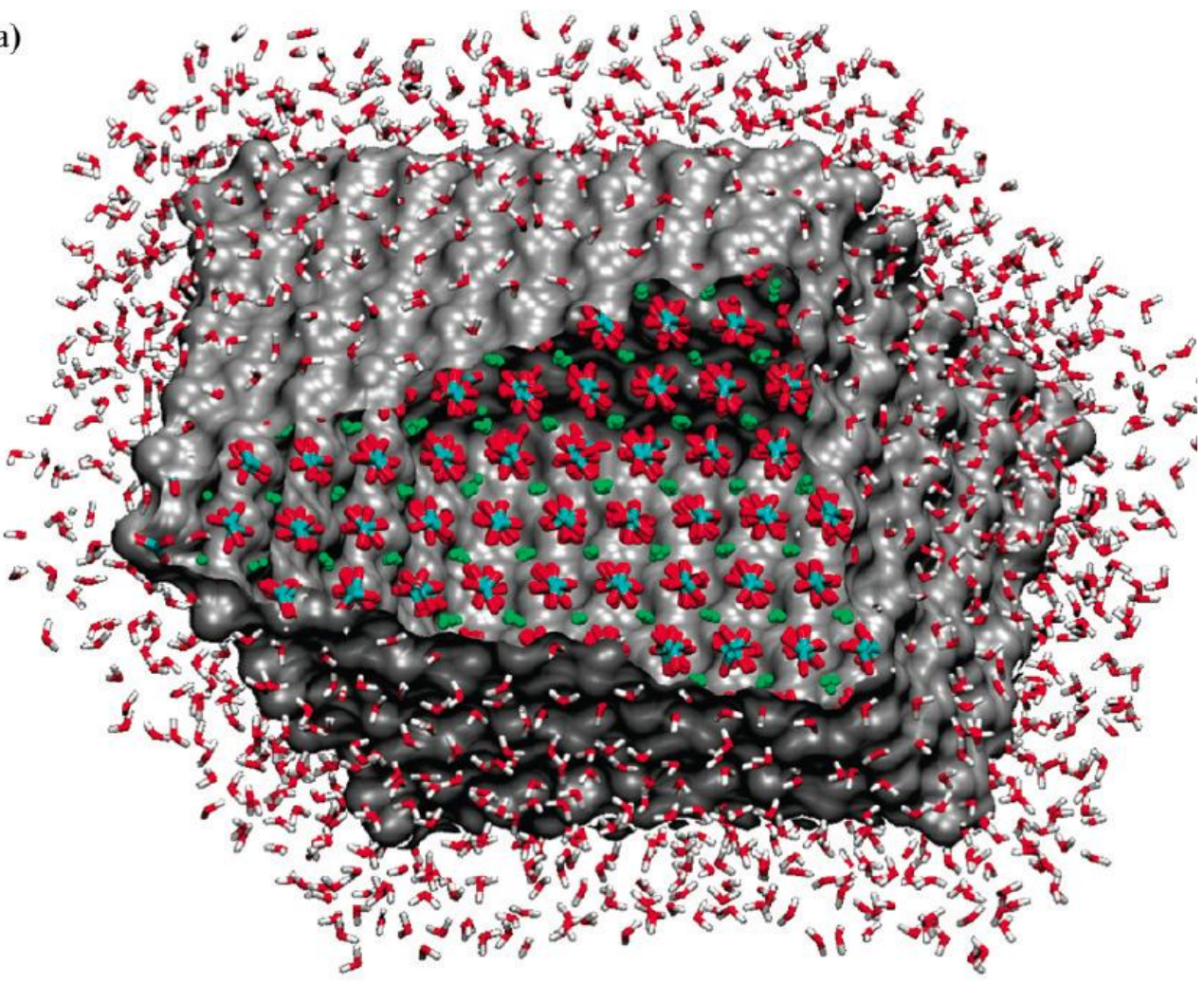

b)

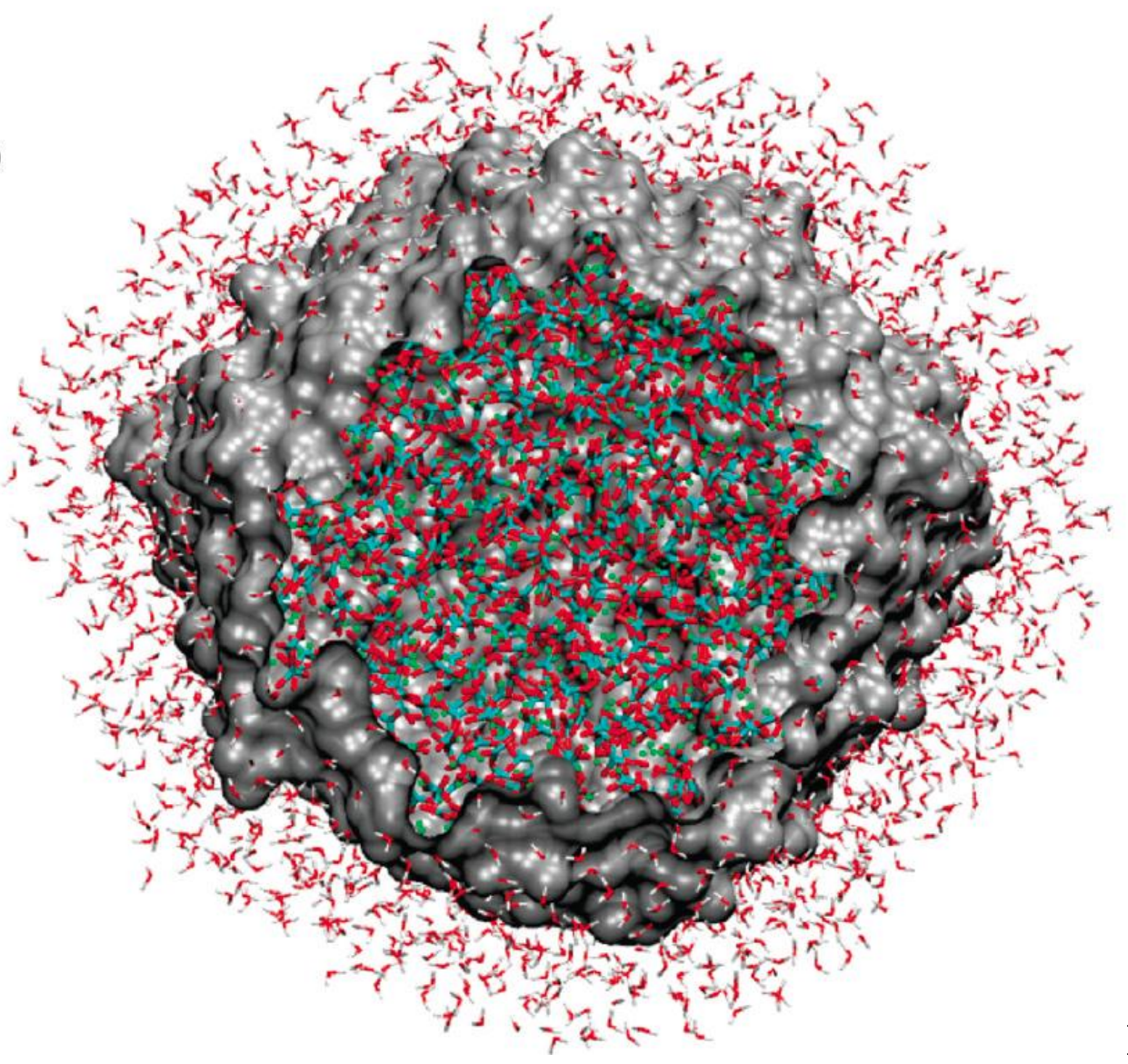

Figure 9 